\title{
Quantitative Trait Locus Analysis in Avocado: The Challenge of a Slow-maturing Horticultural Tree Crop
}

\author{
Vanessa E.T.M. Ashworth ${ }^{5}$ \\ Department of Botany and Plant Sciences, University of California, Riverside, CA 92521
}

Haofeng Chen ${ }^{1}$ and Carlos L. Calderón-Vázquez ${ }^{2}$

Department of Ecology and Evolutionary Biology, University of California, Irvine, CA 92697

Mary Lu Arpaia

Department of Botany and Plant Sciences, University of California, Riverside, CA 92521

David N. Kuhn ${ }^{3}$

USDA-ARS Subtropical Horticulture Research Station, Miami, FL 33158

Mary L. Durbin ${ }^{3}$ and Livia Tommasini ${ }^{4}$

Department of Ecology and Evolutionary Biology, University of California, Irvine, CA 92697

Elizabeth Deyett and Zhenyu Jia

Department of Botany and Plant Sciences, University of California, Riverside, CA 92521

Michael T. Clegg ${ }^{3}$

Department of Ecology and Evolutionary Biology, University of California, Irvine, CA 92697

Philippe E. Rolshausen 5

Department of Botany and Plant Sciences, University of California, Riverside, CA 92521

\begin{abstract}
Additional INDEX words. Persea americana, $\beta$-sitosterol, candidate gene, linkage map, marker-assisted selection, vitamin E
Abstract. The glossy, green-fleshed fruit of the avocado (Persea americana) has been the object of human selection for thousands of years. Recent interest in healthy nutrition has singled out the avocado as an excellent source of several phytonutrients. Yet as a sizeable, slow-maturing tree crop, it has been largely neglected by genetic studies, owing to a long breeding cycle and costly field trials. We use a small, replicated experimental population of 50 progeny, grown at two locations in two successive years, to explore the feasibility of developing a dense genetic linkage map and to implement quantitative trait locus (QTL) analysis for seven phenotypic traits. Additionally, we test the utility of candidate-gene single-nucleotide polymorphisms developed to genes from biosynthetic pathways of phytonutrients beneficial to human health. The resulting linkage map consisted of 1346 markers $(1044.7 \mathrm{cM})$ distributed across 12 linkage groups. Numerous markers on Linkage Group 10 were associated with a QTL for flowering type. One marker on Linkage Group 1 tracked a QTL for $\beta$-sitosterol content of the fruit. A region on Linkage Group 3 tracked vitamin $\mathrm{E}$ ( $\alpha$-tocopherol) content of the fruit, and several markers were stable across both locations and study years. We argue that the pursuit of linkage mapping and QTL analysis is worthwhile, even when population size is small.
\end{abstract}

Avocado is a long-lived tree crop grown worldwide for its tasty and nutritionally valuable fruit. Cultivar Hass dominates the United States market, where production in 2017 ran to 146,000 tons valued at $\$ 392$ million (U.S. Department of Agriculture, 2018). Mexico generated over half of the global

Received for publication 29 Apr. 2019. Accepted for publication 4 July 2019. This research was supported in part by a University of California Discovery Grant and the California Avocado Commission (CAC) to M.C., V.A., C.C., H.C., M.D., and L.T.; by the CAC to M.A.; and USDA-ARS (CRIS 663121000-022) to D.K.

${ }^{1}$ Current address: National Institute for Health and Family Planning, No. 12 Dahuisi Road, Beijing 100081, China.

${ }^{2}$ Current address: Instituto Politécnico Nacional, CIIDIR Unidad Sinaloa, San Joachín, Guasave, Sinaloa, Mexico.

${ }^{3}$ Retired.

${ }^{4}$ Independent researcher.

${ }^{5}$ Corresponding authors. E-mail: vanessa.ashworth@ucr.edu or philippe rolshausen@ucr.edu. output of primarily 'Hass' in 2017, with Peru, Chile, South Africa, Dominican Republic, New Zealand, Israel, and others contributing a substantial market share.

'Hass' has attained its current popularity owing to its excellent flavor, but the cultivar does not excel in all aspects of its growth and productivity, and there is a need to develop new cultivars with improved characteristics. Breeders, therefore, need to consider a wide range of yield- and growth-related attributes that, collectively, ensure efficient and reliable fruit production into the future (Lahav and Lavi, 2009), including tree size and shape, flowering season, and early onset of fruit production, as well as factors contributing to a high fruit set, such as flowering type. Fruit nutritional composition is a further aspect that has seen a recent surge in interest. Among the health benefits attributed to avocado are its heart-healthy properties, reduction of blood lipids, and anticarcinogenic properties (D’Ambrosio, 2007; Ding et al., 2009; Lopez-Ledesma et al., 
1996) conferred by three main groups of compounds: $\beta$-sitosterol, carotenoids, and vitamin E.

Most of these phenotypic traits are inherited in a quantitative fashion; i.e., they are controlled by many genes of small effect and are typically under strong environmental influence. Yet only the genetic component of a phenotype will respond to breeding. Two studies in avocado (Calderón-Vázquez et al., 2013; Chen et al., 2007) used quantitative genetics to tease apart the genetic and the environmental components of the phenotypic value of a suite of quantitative traits. Chen et al. (2007) demonstrated for the progeny of cultivar Gwen that major growth-related traits, such as plant height and trunk- and canopy diameter, were under genetic control and showed sufficient heritability to respond to selection. Similarly, Calderón-Vázquez et al. (2013) showed for a 'Gwen' × 'Fuerte' experimental population - a subset of the population studied by Chen et al. (2007) — that $\beta$-sitosterol, carotenoids, and vitamin $\mathrm{E}$ of the fruit are likely to respond to breeding.

On theoretical grounds, therefore, breeding avocado for growth-related traits and enhanced levels of fruit nutrients is feasible. However, breeding in this long-lived tree crop is frustrated by an outcrossing breeding system, high heterozygosity, long generation times [up to 15 years (Bergh and Lahav, 1996)], and the need for costly field trials to accommodate tree size and a protracted maturation (Van Nocker and Gardiner, 2014). Moreover, controlled pollination is impracticable (Degani et al., 2003; Lammerts, 1942) owing to a profusion of tiny flowers and immature fruitlets - most of which are shed prematurely, and conventional breeding populations (e.g., doubled haploids, recombinant inbred lines) do not exist. At this time, avocado breeders have no option but to use phenotypic selection, which is associated with slow breeding advance. A move toward molecular breeding is a promising alternative to accelerate selection progress and to reduce costs associated with the maintenance of breeding populations.

When designing large-scale experiments leading to molecular breeding, the problem of high land and labor costs loom large, so genetic mapping populations tend to be small and poorly replicated, predisposing data to low statistical power. Yet many horticultural tree crops produce high-value fruit for which the genetic dissection of phenotypic traits is of considerable interest, raising the question whether mapping and quantitative trait locus studies may nonetheless be worthwhile, given adequate precautions. With the advent of next-generation technologies, the costs associated with developing abundant genetic markers have declined significantly, and a shortage of markers no longer represents a constraint. We explore the possibility of generating a linkage map and of estimating QTLs for seven phenotypic traits collected in a mapping population of 50 trees using over 5000 molecular markers. We ask whether a modestly sized mapping population can be used to estimate significant QTL loci and whether these loci are likely to be sufficiently robust.

\section{Materials and Methods}

Mapping POPUlation. The experimental population of avocado trees consisted of the full-sib progeny of a 'Gwen' $(\mathrm{G}) \times$ 'Fuerte' $(\mathrm{F})$ cross. The $\mathrm{G} \times \mathrm{F}$ progeny is a subset of a larger population of open-pollinated trees raised from the fruit of a 'Gwen' maternal tree. Each progeny tree was screened using 10 simple sequence repeat (SSR) markers (Ashworth et al., 2004) to verify the origin of the pollen source. Of more than 200 progeny genotypes analyzed, 50 were the result of the cross $\mathrm{G} \times \mathrm{F}$ and were set aside for the mapping project. The remainder consisted of about 50 individuals each of $G \times$ 'Bacon', $\mathrm{G} \times$ 'Zutano', and a miscellaneous group of largely unidentified pollen origin (Chen et al., 2007) that are not considered further here.

Four clonal replicates of each $\mathrm{G} \times \mathrm{F}$ progeny tree were grafted on 'Duke 7' rootstock and planted at two sites in southern California: two of the four replicate trees were grown in a randomized block design at a coastal location [University of California (UC) South Coast Research and Extension Center, Irvine, CA] and the other two replicate trees at an inland location (Agricultural Operations, UC Riverside campus, Riverside, CA), also in a randomized block layout. Each location, therefore, contained two replicates of 50 tree genotypes (100 trees). All trees were planted in the ground between Fall 2001 and Spring 2003.

Trees were spaced at $6.1 \mathrm{~m}$ between rows and at $4.6 \mathrm{~m}$ between trees within the same row. At the coastal site, fertilizer was applied at $0.45 \mathrm{~kg} /$ tree as a granular formulation of $15 \mathrm{~N}-$ $6.5 \mathrm{P}-12.5 \mathrm{~K}$ in late March/early April. At the inland site, a 32N$0 \mathrm{P}-0 \mathrm{~K}$ fertilizer solution was introduced into the irrigation water at $284.24 \mathrm{~L} \cdot \mathrm{ha}^{-1}$ in January. At both locations, the fertilizer regime was managed to industry standard. Irrigation water was dispensed from two microsprinklers per tree following guidelines established by California Irrigation Management Information System (CIMIS, 2003). The coastal location (Irvine) differed from the inland location (Riverside) by higher average rainfall, cooler average summer temperatures, and warmer average winter temperatures (Table 1). Soils at both locations were sandy loams. The Riverside site followed a gentle hillside contour that consisted of three different sandy loam subtypes (Table 1).

Phenotypic traits. Seven datasets were collected from the experimental trees, including one qualitative (flowering type) and six quantitative (three measures of tree dimension, and three nutrients assayed in the avocado fruit flesh). Descriptive statistics for each quantitative trait are provided in Fig. 1.

Flowering type was recorded in Apr. 2013 at the coastal location in 100 trees. Avocado flowers exhibit protogynous dichogamy, a mechanism designed to prevent self-pollination by temporally separating stigma receptivity and pollen release (Sedgley, 1985). A tree was recorded as having B-type flowering if its flowers were in the male phase in the morning and as having A-type flowering if flowers were in the female phase in the morning. In commercial orchards, optimal pollination and fruit set in cultivars with A-type flowering (e.g., 'Hass' and 'Gwen') is achieved by interplanting with B-type pollinizer cultivars (e.g., 'Fuerte' and 'Bacon') (Alcaraz and Hormaza, 2009). This trait was scored as a discrete character (presence or absence), with A-type flowering recorded as "1" and B-type flowering as " 2 ."

Measures of tree growth were collected at both locations each year from 2003 to 2005, but only the final year's data were used in this study because the later-planted trees were still very immature during the first two years. Three measurements of tree dimension-trunk diameter, tree height, and canopy diameter-were recorded as a way of characterizing the threedimensional aspect of early tree growth (Chen et al., 2007). Trunk diameter was determined at $\approx 10 \mathrm{~cm}$ aboveground in two perpendicular orientations, with values averaged. Plant height 
Table 1. Climatic characteristics at Irvine and Riverside, CA, the two locations of the avocado mapping populations. Data are averages for 19812010 (U.S. Climate Data, 2018).

\begin{tabular}{lrr}
\hline Climate & Irvine & Riverside \\
\hline Annual high temperature $\left({ }^{\circ} \mathrm{C}\right)$ & 22.6 & 26.4 \\
Highest monthly average temperature-August $\left({ }^{\circ} \mathrm{C}\right)$ & 28.3 & 35.0 \\
Annual low temperature $\left({ }^{\circ} \mathrm{C}\right)$ & 12.4 & 10.8 \\
Lowest monthly average temperature-December $\left({ }^{\circ} \mathrm{C}\right)$ & 8.3 & 17.5 \\
Average temperature $\left({ }^{\circ} \mathrm{C}\right)$ & 366.7 & 18.6 \\
Average annual precipitation $(\mathrm{mm})$ & San Emigdio fine & 262.1 \\
Soil type & sandy loam & Arlington fine sandy \\
& & loam; Hanford \\
& & coarse sandy \\
& & loam; Ramona \\
& & sandy loam \\
\hline
\end{tabular}

was measured from ground level to the tip of the tree. Canopy diameter was determined at the widest part of the canopy in two orientations: parallel to the orchard row and perpendicular to the row, with the two values averaged.

Fruit nutrient composition $[\alpha$-tocopherol (the most biologically active form of vitamin $\mathrm{E}$ in humans), $\beta$-sitosterol, and carotenoids] was assayed in fruit collected at both locations in 2009 and 2010. Fruit preparation and chemical assays for determination of the contents of $\alpha$-tocopherol, $\beta$-sitosterol, and carotenoids in fruit tissue were adapted from Jeong and Lachance (2001), Mäeorg et al. (2007), and Ryan et al. (2007) and are detailed in Calderón-Vázquez et al. (2013). For any given tree, five fruit were picked at an optimum dry weight of $20 \%$ and then allowed to ripen in the laboratory. At ripeness, the flesh from the five fruit was pooled and homogenized, and aliquots were frozen and set aside for further analyses. Total carotenoids, which include $\alpha$-carotene, $\beta$-carotene, $\beta$-cryptoxanthin, lutein, and zeaxanthin, were isolated using two extractions in hexane/petroleum ether $(1: 1)$. An aliquot of the resulting aqueous phase was analyzed by taking a spectrophotometric reading at $456 \mathrm{~nm}$ and comparing it to a standard curve for $\beta$-carotene (C4582; Sigma-Aldrich, St. Louis, MO) according to Luterotti et al. (2006). Beta-sitosterol and $\alpha$-tocopherol contents were determined by application of the organic phase fraction to thin-layer chromatographic plates. Bands were visualized by dipping in phosphomolybdic acid (02553, Sigma-Aldrich) and quantified on an AlphaImager HP System (ProteinSimple, Santa Clara, CA) using standard curves generated from reference samples $[\beta$-sitosterol (S1270, SigmaAldrich), $\alpha$-tocopherol (T3251, Sigma-Aldrich)]. Values for the parental cultivars Gwen and Fuerte were determined in trees growing at the coastal location using the same preparation and assay conditions as for the progeny (Calderón-Vázquez et al., 2013).

Statistical analyses of the phenotypic data were performed in $\mathrm{R}$ version 3.4.4 ( $\mathrm{R}$ Core Team, 2019) using a nonparametric Kruskal-Wallis test to compare datasets, followed by a Wilcox test for pairwise comparisons and calculation of probability values.

GeNeTIC MARKERS. The genetic markers implemented in this study consisted of SSRs and single-nucleotide polymorphisms (SNPs) from several sources; the bulk of markers were SNPs developed by Kuhn et al. (2019). In our map, these SNPs were used to augment the total number of markers to ensure adequate map density. The second set of SNP markers was developed in a gene discovery effort targeting candidate genes from several biosynthetic pathways involved in fruit nutrient composition. These candidate-gene SNPs (CG-SNPs) have not previously been published and their development is described in the following two paragraphs. In addition, we used published SSR markers developed by Sharon et al. (1997), Borrone et al. (2007), and Ashworth et al. (2004), as well as 28 SSR markers available from GenBank (V.E. Ashworth, C. Calderón-Vázquez, M.L. Durbin, L. Tommasini, and M.T. Clegg, unpublished data).

SNPs by Kuhn et al. [2019 (FL-SNPs)] originated by Illumina GAII sequencing (Illumina, San Diego, CA), and the individuals of our 'Gwen' $\times$ 'Fuerte' mapping population were included on the Illumina Infinium oligonucleotide array chip that assayed each tree genotype for 5050 FL-SNP markers. Details of marker development are provided in Kuhn et al. (2019).

Nutrient-related candidate genes were identified by aligning avocado expressed sequence tag (EST)/cDNA (complementary DNA) sequences from fruit-, flower-, and other organ-specific libraries developed by Cornell University [Ithaca, NY (Floral Genome Project, 2005)], HortResearch (Mt Albert, New Zealand), and CINVESTAV (Irapuato, Mexico) to sequences of functionally characterized gene sequences deposited in TAIR (2005) or NCBI (2005). Avocado mRNA sequences showing high similarity to core enzymes in the flavonoid, carotenoid, fatty acid, and B-, C-, and E-vitamin biosynthesis pathways were retained. Their relevance in determining fruit nutritional composition was further verified by comparison with sequences from an avocado cDNA library developed from the fruit of cultivar Hass. Sequence alignment allowed design of amplification primers in conserved regions. Nested sequencing primers provided about 500 base pairs of high-quality DNA sequence.

SNP discovery was performed in sequences from a panel of 10 randomly chosen 'Gwen' $\times$ 'Fuerte' progeny genotypes. SNPs were identified by standard resequencing using the Sanger method. Sequence reads were assembled using Phred/ Phrap/Consed (Ewing and Green, 1998; Gordon et al., 1998), and PolyPhred was used to detect the SNP sites (Nickerson et al., 1997). A total of 83 SNPs was developed from 28 candidate genes. Avocado genomic DNA of the 10 'Gwen' $\times$ 'Fuerte' progeny was extracted from frozen young (flushing) leaves using the DNeasy Plant Mini Kit (Qiagen, Valencia, CA). Forward and reverse reads were generated during the sequencing phase. Sequences from the SNP phase were 

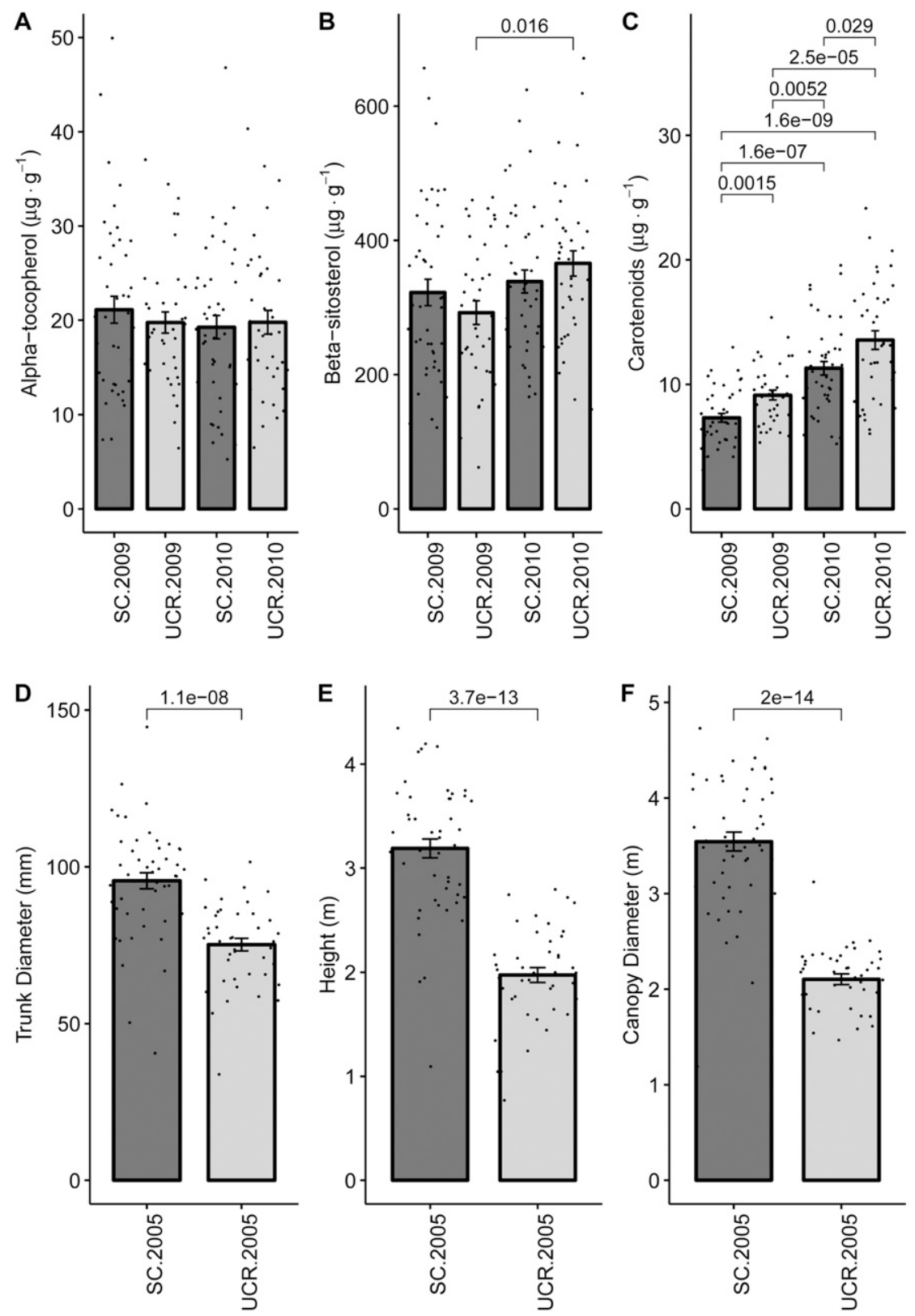

Fig. 1. Variance statistics for six quantitative traits determined in an avocado mapping population growing at two locations in southern California [South Coast Research \& Extension Center in Irvine, CA (SC) and Agricultural Operations of the University of California at Riverside (UCR)]. Dots represent samples, bars show means and SE. Numbers above brackets are probability values (no brackets are shown for $P>0.05$ ).

(2007; 13 markers), and Ashworth et al. (2004; 39 markers). Twentyeight new SSR markers are detailed in Supplemental Table 2; their development and assay conditions are identical to those given in Ashworth et al. (2004). SSR markers of Borrone et al. (2007) were developed from ESTs.

SSR markers originating at UC (CA-SSRs) were prefixed with AVO, AUCR, or AVD if developed from a genomic library enriched for dinucleotide repeats; a prefix of AVT denotes development from a trinucleotide-enriched genomic library (Ashworth et al., 2004). SSR markers developed by Borrone et al. (2007; FL-SSRs) are prefixed with SHRSPa (Subtropical Horticulture Research Station-Persea americana) followed by a three-digit number. AVMIX3 originated from Sharon et al. (1997). CG-SNPs are abbreviated in relation to the candidate gene name and numbered sequentially based on the SNP position within the gene sequence. The FL-SNPs (Kuhn et al., 2019) are prefixed by SHRSPaS00, followed by SNP numbers in the range 1000 to 6999. All CG-SNPs from the same candidate gene were retained unless a SNP showed strong segregation distortion or many missing data.

LiNKAGE MAP CONSTRUCTION. OUR linkage map [henceforth "California (CA)"-map] was generated using the regression mapping algorithm implemented in JoinMap version 4 (Van Ooijen, 2006) that allows analysis of a mixed set of marker types and segregation patterns. Population type was set to cross pollination (CP). We used regression mapping combined with the Kosambi function of transform-

sequenced only in one direction (either $5^{\prime}$ to $3^{\prime}$ or $3^{\prime}$ to $5^{\prime}$ ), either using polymerase chain reaction (PCR) amplification primers or nested primers (Supplemental Table 1). PCR amplification conditions were as follows: preheating at $94{ }^{\circ} \mathrm{C}$ for $2 \mathrm{~min}$, then 35 cycles of $94^{\circ} \mathrm{C}$ for $30 \mathrm{~s}$, annealing at primerspecific temperatures $\left(47\right.$ to $58^{\circ} \mathrm{C}$ ) for $30 \mathrm{~s}$ and extension at $72{ }^{\circ} \mathrm{C}$ for between $30 \mathrm{~s}$ and $1 \mathrm{~min} 45 \mathrm{~s}$, ending with a final extension of $72^{\circ} \mathrm{C}$ for $5 \mathrm{~min}$. PCR products were purified using the QIAquick PCR purification kit (Qiagen) or ExoSAP-IT (USB-Affymetrix, Cleveland, $\mathrm{OH}$ ). Sequencing products were run on a DNA sequencer (Applied Biosystems 3730xl DNA Analyzer; Thermo Fisher Scientific, Waltham, MA).

SSR markers included 53 published markers. They were sourced from Sharon et al. (1997; 1 marker), Borrone et al. ing recombination frequencies into map units (centiMorgans). A log-of-odds (LOD) value of 5.0 was used for linkage group selection. MapChart version 2 (Voorrips, 2002) enabled markers to be graphically represented on their corresponding linkage group (LGs) based on the map distances determined via linkage analysis.

The chi-squared test implemented in Joinmap (Van Ooijen, 2006) was used to examine each marker for segregation distortion. Although distorted markers can be the cause of Type 1 Error (detecting false linkage), only markers with values of 8 or higher were pruned from the dataset, as modest amounts of segregation distortion are thought to contribute pertinent information (Hackett and Broadfoot, 2003; Wang et al., 2005). 
To explore whether missing data may be affecting marker distribution and distances when working with small mapping populations, we developed a second map from which all markers with missing data had been removed. Additionally, we compared the CA-map to a high-density map integrated from four reciprocal mapping populations [514 progeny of 'Tonnage' $\times$ 'Simmonds', 249 of 'Simmonds' $\times$ 'Tonnage', 346 of 'Hass' $\times$ 'Bacon', and 230 of 'Bacon' $\times$ 'Hass'; henceforth "FL-map" (Rendón-Anaya et al., 2019)] that included the same set of 5050 next-generation SNPs (Kuhn et al., 2019). The comparison was made using the VLOOKUP function in Excel (version 16.16.1; Microsoft, Redmond, WA) to check for marker distribution across and within linkage groups for markers common to both maps.

QTL ANALYSIS. QTL analysis was performed using both interval mapping [IM (Lander and Botstein, 1989)] and nonparametric mapping [Kruskal-Wallis (KW) test; Kruskal and Wallis (1952)] implemented in MapQTL version 5 (Van Ooijen, 2004). Under IM, QTL significance was assigned to a marker locus in relation to the LOD likelihood scores determined using 1000 permutations of the data at a significance level of $P=0.05$. In the maximum likelihood mixture model of IM, where LOD scores are calculated using an iterative algorithm, an iteration number of 20 was used as a cut-off to declare a significant QTL, with values above 20 representing a poor fit of the data to the model (Van Ooijen, 2004). Markers exceeding the cutoff of 20 for iteration number were disregarded. The $\mathrm{KW}$ test evaluates each marker independently regardless of its location on the linkage map. It is recommended for data that are not normally distributed, such as qualitative data, counts, data with outliers, and truncated data probabilities (Kruglyak and Lander, 1995), and it assigns significance in relation to the test statistic $\mathrm{K}^{*}$, with a value of $P \geq 0.005$ (denoted as $* * * *$ in MapQTL) considered sufficiently stringent to declare a marker as being significantly associated with a QTL.

To verify significant QTLs, we performed an approximation of the multiple-QTL model (MQM) by manually selecting markers located close to a QTL as cofactors. The MQM model is more accurate and efficient at detecting QTLs than IM because the latter ignores the effects of other QTLs, but MQM suffers from being computationally intensive. A work-around was developed by Jansen (1993) and is implemented in MapQTL in the "rMQM" module. However, owing to the small population size and heterogeneously heterozygous population type ("CP" in MapQTL) of this dataset, we were not able to take advantage of the Automatic Cofactor Selection analysis available in MapQTL to perform backward elimination because it uses many degrees of freedom (df) and is computationally too demanding. Instead, we manually chose cofactors guided by the output from IM, sequentially selecting markers closest to a significant QTL and running rMQM. QTLs were retained if successive exclusion of cofactors did not alter the LOD values associated with the QTL.

Where multiple datasets were available, MapQTL analyses were performed for each location (coastal or inland) separately in the case of the growth-related traits (trunk diameter, plant height, and canopy diameter), as previous studies had shown significant location effects (Chen et al., 2007). For fruit nutrient content, analyses were also run on separate datasets ( 2 years and two locations) because Calderón-Vázquez et al. (2013) had demonstrated significant effects of harvest year on the contents of two of the three nutrients and a significant location effect on carotenoid contents, as well as interaction effects for genotype $\times$ environment $(\beta$-sitosterol and carotenoids) and genotype $\times$ year $(\beta$-sitosterol). Flowering was analyzed for a single year at the coastal location.

In all cases, we examined the output from both IM and the non-parametric KW test to declare significant QTLs, emphasizing those markers that were endorsed by both algorithms. Consideration of both the IM and KW output was deemed prudent (Kruglyak and Lander, 1995), given that the small population size $(n=50)$ may have affected the accuracy or power of the algorithms.

\section{Results}

Phenotypic TRaits. Plots showing the distribution of tree measurements at both locations and of the fruit nutrient data at all four location/year combinations are presented in Fig. 1. Trees were consistently somewhat shorter at Riverside than at Irvine, averaging $1.97 \pm 0.466$ and $3.19 \pm 0.639 \mathrm{~m}$, respectively. Trees at Riverside also developed smaller canopies $(2.1 \pm 0.368$ and $3.55 \pm 0.691 \mathrm{~m}$, respectively) and trunk diameters $(75.13 \pm$ 13.1 and $95.53 \pm 18.0 \mathrm{~mm}$, respectively).

Values of the three fruit nutrients responded differently depending on environment and year; $\alpha$-tocopherol values were not significantly different for either year or location. Betasitosterol values were significantly different between years at the Riverside location, with higher values occurring in 2010. Differences between years at the Irvine location were not significant. Carotenoid contents were significantly different for all location/year comparisons, with values significantly higher at Riverside than at Irvine and significantly higher in 2010 than in 2009.

One genotype consistently produced fruit with the highest $\alpha$-tocopherol concentrations at Irvine in both years and at Riverside in 2010 but failed to produce any fruit at Riverside in 2009 , leading to a missing data point. The same genotype was also responsible for the highest $\beta$-sitosterol values at Irvine and Riverside in 2010 and the second-highest value in Irvine in 2009 . In both years, almost half the progeny in Irvine exceeded $\alpha$-tocopherol contents measured in the parental cultivars [19.5 and $19.0 \mu \mathrm{g} \cdot \mathrm{g}^{-1}$ fresh weight (FW) in 'Gwen' and 'Fuerte', respectively]. Two genotypes exceeded the value of their maternal parent more than 2-fold. Progeny values varied more than 6-fold (2009) and 8-fold (2010) at Irvine and more than 5-fold (2009) and 6 -fold (2010) at UCR.

For $\beta$-sitosterol, values of the male parent $\left(672 \mu \mathrm{g} \cdot \mathrm{g}^{-1} \mathrm{FW}\right)$ consistently exceeded values in the progeny; but seven and five progeny genotypes, respectively, exceeded the value in 'Gwen' $\left(469 \mu \mathrm{g} \cdot \mathrm{g}^{-1} \mathrm{FW}\right)$ in 2009 and 2010 . Progeny values varied more than 5-fold (2009) and 4-fold (2010) at Irvine and more than 7fold (2009) and 4-fold (2010) at UCR.

Carotenoid contents were higher in 'Fuerte' $\left(9.8 \mu \mathrm{g} \cdot \mathrm{g}^{-1} \mathrm{FW}\right)$ than in 'Gwen' (8.37 $\left.\mu \mathrm{g} \cdot \mathrm{g}^{-1} \mathrm{FW}\right)$. In 2009 and 2010, eight and 27 progeny genotypes, respectively, exceeded 'Fuerte' values. Values in the progeny varied 4-fold (2009) and 3-fold (2010) in Irvine and 3-fold (2009) and almost 4-fold (2010) at UCR.

Flowering type was determined at Irvine for 47 genotypes for which two replicate trees were available, 31 genotypes showing B-type flowering (as in 'Fuerte'), and 16 showing Atype flowering (as in 'Gwen'). All replicate pairs showed the same flowering type. 
LiNKAGE MAPPING. We pre-screened 5050 FL-SNPs developed by Kuhn et al. (2019) to eliminate markers that were invariant or uninformative in the parental genotypes 'Gwen' and 'Fuerte'. The remaining FL-SNP markers (2608) were then combined with 146 informative SNP and SSR markers; 83 SNPs developed to eight candidate genes of nutritional pathways and 63 SSR markers. In total, 2754 markers were imported into a JoinMap version 4.0 (Van Ooijen, 2006) data matrix for linkage mapping, of which 1346 markers (49\%) placed on 12 linkage groups at a LOD value of 5.0, constituting the CA-map (Supplemental Fig. 1).

A total of 1399 markers were eliminated because of identical segregation or because of strong segregation distortion (38 markers with $\chi^{2}=8.00-31.04, P=0.01-0.0000001$, df $\left.=1-3\right)$. The placed markers consisted of 1235 FL-SNPs $(91.8 \%), 58$ CG-SNPs (4.3\%), and 53 SSR markers [AVMIX3, 13 FLSSRs, and 39 CA-SSRs (3.9\%)]. Of the 1346 markers on the map, $616(45.8 \%)$ were heterozygous in both parents, of which six segregated with four alleles (SSRs), 20 with three alleles (SSRs), and 590 with two alleles (SNPs and SSRs). Markers segregating in only one of the parents $(730 ; 54.2 \%)$ numbered 309 in 'Gwen' and 421 in 'Fuerte'.

Marker number per linkage group averaged 112, ranging from 56 loci (LG12) to 207 loci (LG2). Combined linkage group length was $1044.7 \mathrm{cM}$, ranging from $61.483 \mathrm{cM}$ on LG2 to $121.125 \mathrm{cM}$ on LG3, and averaging $87.06 \pm 19.77 \mathrm{cM} /$ linkage group. The mean number of loci/cM was 1.32 . Gaps larger than $5 \mathrm{cM}$ occurred on four linkage groups. The densest linkage group was LG2 (3.37 loci/cM). Sparse coverage characterized distal portions of LG7 (Supplemental Fig. 2). Supplemental Table 3 shows marker order on the 12 avocado linkage groups obtained in this study.

An exploratory map made up exclusively of markers containing no missing data closely resembled the CA-map. Also using a LOD value of 5.0 to assign markers to linkage groups, this map contained 1238 markers on 12 linkage groups with a combined length of $1036.3 \mathrm{cM}$. Linkage groups averaged 103 loci and $86.35 \pm 27.44 \mathrm{cM}$. Of the 1238 placed markers, one SSR marker segregated with four alleles, four SSRs segregated with three alleles, 555 were of JoinMap segregation type $h \mathrm{k} \times \mathrm{hk}, 289$ of type $1 \mathrm{~m} \times 1 \mathrm{l}$, and 389 of type nn×np.

Comparison of the CA-map with the highly saturated FLmap (Rendón-Anaya et al., 2019) showed excellent agreement between the two maps, as markers common to both maps were assigned to the same linkage group and marker order was comparable (Supplemental Fig. 2). Although a few linkage groups showed inverted segments (Supplemental Fig. 2), we did not adopt the FL-map marker order. FL-map linkage groups contained $\approx 2.0$ to 3.3 times as many marker loci as their CAmap counterparts. Overall, the number of loci on the FL-map was about 2.6 times greater than that on the CA-map, and total linkage group length (cM) of the FL-map was 1.73 times greater. The average marker density for the FL- and CA-maps was 1.97 and 1.32 markers/cM, respectively.

Of the 58 CG-SNPs assigned to a linkage group, the greatest number (13 SNPs; 22.4\%) mapped to LG2. SNPs of the same candidate gene always mapped to the same linkage group. In most cases SNPs from the same candidate gene mapped in close proximity. Exceptions were the SNPs of CUT1 (12.569 cM apart), MEP (8.119 cM apart), PSY (6.731 cM apart), and VTE1_687 (6.015 cM from the nearest SNP, VTE1_573).
QTL ANALYSIS. The number of markers showing a significant association (based on KW and IM) with each of the seven phenotypic traits is summarized in Table 2. IM failed to identify any markers associated significantly with canopy diameter, tree height, or trunk diameter at either location. KW identified five significant markers for trunk diameter and three for canopy diameter at Irvine and a single significant marker for canopy diameter and tree height at Riverside.

The content of total carotenoids in the fruit did not show significant association with any marker based on IM (Table 2). Based on KW, significant QTLs were located on LG1, 3, and 6.

QTL analysis of fruit $\beta$-sitosterol content at Riverside in 2010 revealed one marker (SHRSPaS006673) at $61.087 \mathrm{cM}$ on LG1 to be significantly associated using IM at a LOD of 3.72 (Fig. 2), explaining 35.6\% of the variance (Table 2). This marker also achieved significance in the $\mathrm{KW}$ analysis in the same location and year, and at Irvine in 2009 (Table 2). Marker SHRSPaS001205 (LG1), less than $2 \mathrm{cM}$ away from SHRSPaS006673, was also significantly associated with $\beta$-sitosterol content at Irvine in 2009 and Riverside in 2010, based on KW analysis. Figure 2 compares the IM LOD profiles of markers on LG1 for $\beta$-sitosterol in all four datasets (Irvine and Riverside in 2009 and 2010).

In IM analyses, markers on LG3 were significantly associated with $\alpha$-tocopherol content at Irvine in both years - 12 in 2009 and 15 in 2010 - achieving LOD values of up to 4.52 and 4.61 , respectively, and explaining up to $37.7 \%$ and $38.3 \%$ of the variance, respectively (Table 2). No marker attained significance based on IM at Riverside in 2009. Two markers, SHRSPa001282 and SHRSPa003314, were declared significant at both locations and in both years, based on IM and/or KW. Significant QTLs resided on the proximal end of LG3 at 7.968 to $18.601 \mathrm{cM}$ (IM) and at 0 to $27.638 \mathrm{cM}$ (KW; Fig. 2). Three HPT1 CG-SNPs were declared significant based on KW only (Supplemental Table 3).

Flowering type showed significant association with many markers under IM, with LOD values far exceeding the permutation-based thresholds for significance. IM showed a significant association with 45 markers, all of which resided on LG10 (Table 2; Supplemental Table 3; Fig. 2). Twenty-four markers on LG10 exceeded the genome-wide LOD threshold of 7.1 and explained $50.4 \%$ to $100 \%$ of the variance in flowering type. Six of these markers achieved LOD scores of 99.99 in IM and explained $100 \%$ of the variance-but were disregarded because they did not track phenotypic values and represented an artifact of the IM maximum likelihood algorithm applied to non-normal (discrete) data (Van Ooijen, 2009). A further 21 markers on LG10 exceeded the LG-specific LOD threshold, including the CG-SNP DXPS1_1593. All markers on LG10 declared significant at the genome-wide cutoff were located between 26.808 to $53.308 \mathrm{cM}$ (Supplemental Table 3; Fig. 2). Eight of the 24 markers exceeding the genome-wide threshold under IM received no support in the KW test, including the six markers with a 99.99 LOD score. KW analysis identified 22 markers associated significantly with flowering type (Table 2), all but one also residing on LG10: a single QTL-associated marker, SHRSPaS003811, located to LG6 (Supplemental Table $3)$. The two highest-scoring markers in the KW test had $\mathrm{K}^{*}$ values of 38.251 (SHRSPaS001390 and SHRSPaS004380) and were declared significant at $P=0.00001$. Their validity as QTLs was endorsed by IM, which assigned LOD values of 18.66 and 18.34, respectively. Among the markers associated significantly 
Table 2. Evaluation of quantitative trait loci (QTLs) identified by interval mapping (IM) or Kruskal-Wallis analysis (KW) implemented in MapQTL version 5 (Van Ooijen, 2004) for avocado mapping populations growing at two locations in southern California (Irvine and Riverside). Comparisons are made for all markers declared to be significant under the interval mapping (IM) or Kruskal-Wallis (KW) algorithms. Column headings details are as follows. IM = the number of significant loci declared by IM; in parentheses is the percentage of the variance explained by the locus with the highest log-of-odds (LOD) score. KW = the number of significant loci with a significance of **** or higher, based on KW. LGs-IM = the number of different linkage groups (LGs) from which significant markers were drawn, based on IM. LGs$\mathrm{KW}=$ the number of different LGs from which significant markers were drawn, based on KW. QTL $\geq$ two environments $=$ the number of QTLs present in at least two environments (two locations and 2 years for nutrients; two locations for tree measurements).

\begin{tabular}{|c|c|c|c|c|c|c|}
\hline Nutrient & Location, yr & IM [no. $(\%)]$ & KW (no.) & LGs-IM (no.) & LGs-KW (no.) & $\begin{array}{l}\mathrm{QTL} \geq \text { two } \\
\text { environments }\end{array}$ \\
\hline \multirow[t]{4}{*}{ Alpha-tocopherol } & Irvine, 2009 & $12(37.7)$ & 21 & 1 & 3 & $21(5,2)^{z}$ \\
\hline & Irvine, 2010 & $15(38.3)$ & 24 & 1 & 4 & \\
\hline & Riverside, 2009 & $0(39.5)$ & 11 & $\mathrm{n} / \mathrm{a}$ & 3 & \\
\hline & Riverside, 2010 & $0(37.4)$ & 14 & $\mathrm{n} / \mathrm{a}$ & 1 & \\
\hline \multirow[t]{4}{*}{ Beta-sitosterol } & Irvine, 2009 & $0(34.8)$ & 17 & $\mathrm{n} / \mathrm{a}$ & 1 & 11 \\
\hline & Irvine, 2010 & $0(33.9)$ & 6 & $\mathrm{n} / \mathrm{a}$ & 1 & \\
\hline & Riverside, 2009 & $0(35.0)$ & 5 & $\mathrm{n} / \mathrm{a}$ & 2 & \\
\hline & Riverside, 2010 & $1(35.6)$ & 12 & 1 & 3 & \\
\hline \multirow[t]{4}{*}{ Carotenoids } & Irvine, 2009 & $0(28.5)$ & 1 & $\mathrm{n} / \mathrm{a}$ & 1 & 1 \\
\hline & Irvine, 2010 & $0(31.4)$ & 3 & $\mathrm{n} / \mathrm{a}$ & 2 & \\
\hline & Riverside, 2009 & $0(35.8)$ & 8 & $\mathrm{n} / \mathrm{a}$ & 1 & \\
\hline & Riverside, 2010 & $0(35.4)$ & 3 & $\mathrm{n} / \mathrm{a}$ & 2 & \\
\hline \multirow[t]{2}{*}{ Trunk diameter } & Irvine, 2005 & $0(26.6)$ & 5 & $\mathrm{n} / \mathrm{a}$ & 3 & 0 \\
\hline & Riverside, 2005 & $0(25.3)$ & 0 & $\mathrm{n} / \mathrm{a}$ & $\mathrm{n} / \mathrm{a}$ & \\
\hline \multirow[t]{2}{*}{ Canopy diameter } & Irvine, 2005 & $0(26.3)$ & 3 & $\mathrm{n} / \mathrm{a}$ & 2 & 0 \\
\hline & Riverside, 2005 & $0(34.1)$ & 1 & $\mathrm{n} / \mathrm{a}$ & 1 & \\
\hline \multirow[t]{2}{*}{ Height } & Irvine, 2005 & $0(33.9)$ & 0 & $\mathrm{n} / \mathrm{a}$ & $\mathrm{n} / \mathrm{a}$ & 0 \\
\hline & Riverside, 2005 & $0(31.8)$ & 1 & 0 & 1 & \\
\hline Flowering type & Irvine, 2013 & $45(24)^{\mathrm{y}}(100.0)$ & 22 & 1 & 2 & $\mathrm{n} / \mathrm{a}$ \\
\hline
\end{tabular}

${ }_{\mathrm{Z}}^{\mathrm{z}}$ In parentheses: number of QTLs shared by three and four environments, respectively.

$\mathrm{y}_{24}$ QTLs for flowering type were declared significant using the genome-wide permutation threshold [18 after elimination of 6 QTLs with artifactually high LOD values (Van Ooijen, 2009)] and 45 using the linkage-group specific threshold (39 after adjusting for artifactual LOD values).

with flowering type was one SSR marker (AVD010; Supplemental Table 3).

\section{Discussion}

Despite the limited statistical power associated with small sample sizes, this study provided useful mapping information on two important phenotypic traits: flowering type and vitamin E ( $\alpha$-tocopherol) content of the fruit.

Flowering type is not a quantitative trait, and Lavi et al. (1993) suggested control by several loci with several alleles at each locus. A closer look at our data for flowering type uncovered a one-gene Mendelian model that likely governs this important trait in avocado. Using the 13 top-scoring loci on LG10 endorsed by both IM and KW, pairwise analysis showed that they were highly correlated with one another, suggesting a single causal locus with flanking loci linked through linkage disequilibrium (LD). Moreover, 29 (100\%) individuals with genotype "ll" had B-type flowering, whereas-among individuals with genotype "lm" $-16(89 \%)$ individuals had Atype flowering and $2(11 \%)$ individuals had B-type flowering. These results indicate that " $\mathrm{m}$ " is the dominant allele while " 1 " is the recessive allele. The two individuals with genotype "lm" showing the unexpected phenotype likely reflect the effect caused by a gene $x$ environment interaction, which may reduce the penetrance of the dominant trait. This assumption is well supported by Sedgley and Annells' findings (1981), which indicated that avocado flowering was affected by cold temperature, allowing the male and female phases of the flower to overlap. Elucidation of the genes determining flowering type would provide greater flexibility to growers in their choice of pollinizer cultivars.

Alpha-tocopherol content exhibited moderate to high heritability in quantitative genetic analyses (Calderón-Vázquez et al., 2013; Chen et al., 2007) and might be expected to yield some success in breeding programs. The current mapping studies suggest that the variation underlying flowering type and $\alpha$-tocopherol may be the result of mutations at a single genetic locus. A third trait ( $\beta$-sitosterol content of the fruit), also with a substantial heritability (Calderón-Vázquez et al., 2013), provided promising, although not entirely consistent, evidence for a particular chromosomal location.

Not surprisingly, traits of low to moderate heritability do not give consistent results in the QTL analyses, as is the case for plant height, canopy diameter, and trunk diameter [broad-sense heritability estimates in the low- to medium range ( 0.266 to 0.366; Chen et al., 2007)]. Variation underlying these morphological traits is likely to be controlled by many loci throughout the genome and to be subject to substantial environmental variation. So, the failure to map variants associated with these traits is to be expected. Moreover, the high positive correlations between these three measurement traits (Chen et al., 2007) suggest that breeding for tree architecture may not be straightforward. The fact that QTL analysis for these three growth traits 


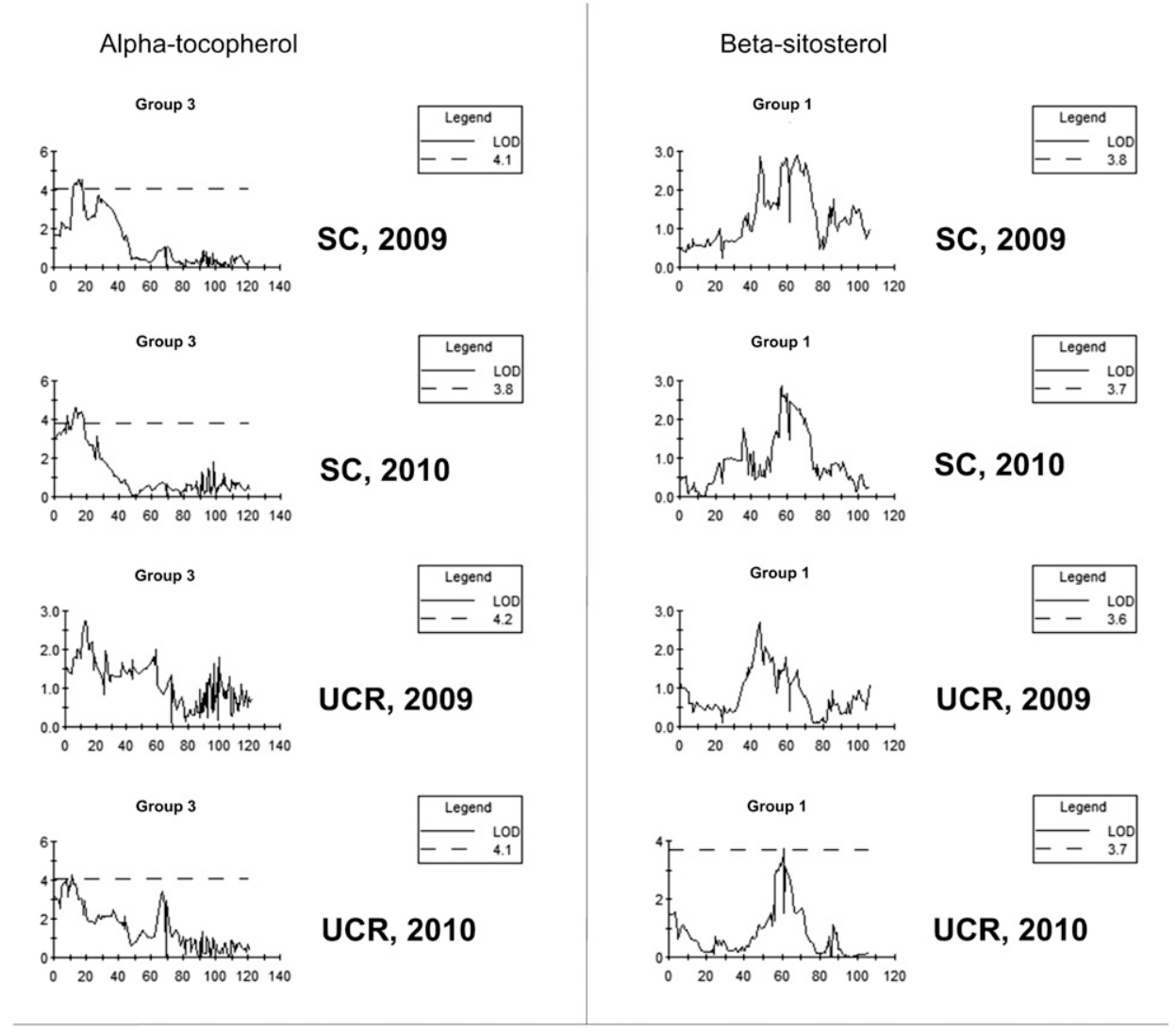

Flowering type

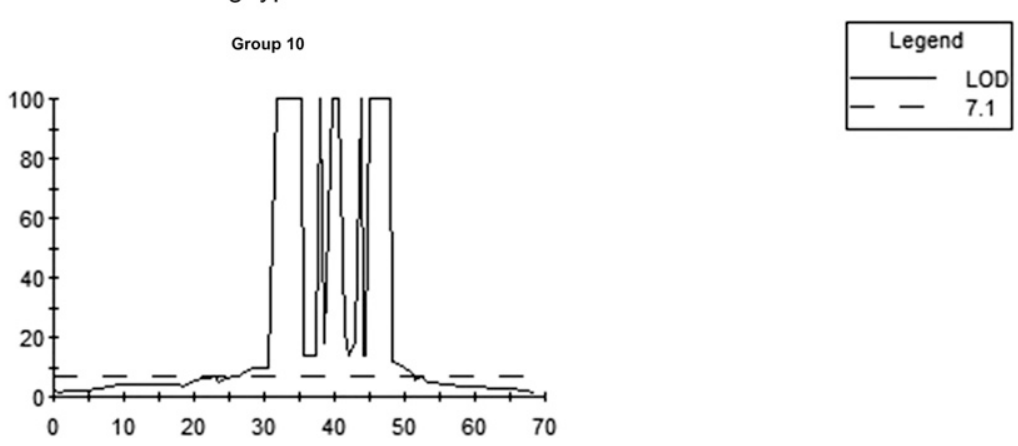

Fig. 2. Plots charting the log-of-odds (LOD) values of markers significantly associated with avocado fruit $\alpha$-tocopherol contents on linkage group (LG) 3 , $\beta$-sitosterol contents on LG1, and flowering type on LG10. For $\beta$-sitosterol and $\alpha$-tocopherol, separate LOD plots are shown for each of 2 years and two locations studied [South Coast Research \& Extension Center in Irvine, CA (SC) and Agricultural Operations of the University of California at Riverside (UCR)]. $X$-axes show map positions (cM).

revealed few significant QTLs under KW analysis (and none under IM) suggests that marker-assisted selection (MAS) for these growth-related traits is not worthwhile.

Nutritional TRAITs. Appreciable genetic determination of the fruit nutrient phenotypes was shown by Calderón-Vázquez et al. (2013), who determined broad-sense heritability for $\alpha$-tocopherol, $\beta$-sitosterol, and carotenoids to be $0.76,0.61$, and 0.47 , respectively. Considerably higher values than those of the tree measurements, these values are consistent with the fact that nutritional traits are the outcome of specific biochemical pathways. Additionally, correlations among the three nutritional traits were low, the highest arising between $\alpha$-tocopherol and $\beta$-sitosterol at $\mathrm{R}=32 \%$ (Calderón-Vázquez et al., 2013). Low correlation also may be due to the discrete biochemical pathways underlying the biosynthesis of these nutrients and will facilitate independent breeding. Significant genotype effects were found for all three nutritional traits (Calderón-Vázquez et al., 2013), but for the other variance components (year, location, and interaction effects), each nutrient responded differently. Combined with the current results, these findings argue that a focus on nutritional/biochemical traits can be effective, despite limited population sizes.

Among the nutrient data, few QTLs performed well across all four environments (two locations and 2 years). Significant QTLs for carotenoid and $\beta$-sitosterol contents were never shared by more than two environments ( 1 and 11 QTLs, respectively, were shared by 2 environments; Table 2). Of 21 QTLs for $\alpha$-tocopherol that were common to at least two environments, five were present in three environments, and two were present in all four environments (Table 2). The discovery of QTL loci that tracked nutrient content across multiple environments is encouraging and presumably reflects genes with stable expression under different environmental conditions.

For $\beta$-sitosterol, the QTL achieving significance at Riverside in 2010 did not stand out in the other year/location combinations, calling into question whether this QTL will be amenable to MAS. It is worth noting, however, that this sole significant marker on LG1 was located adjacent (within 0.49 $\mathrm{cM})$ to an EST-derived FL-SSR marker (SHRSPa102; Supplemental Table 3) that had a very low LOD value in most IM datasets, suggesting SHRSPa102 may not have been correctly placed on the CA-map (Van Ooijen, 2006). The position is visible as an abrupt deep incision on the LOD graph (Fig. 2). It is conceivable that the proximity of an incorrectly placed marker affected the LOD value within the interval surrounding the significant QTL.

Population size considerations. As noted earlier in the section on QTL analysis, one aspect of this study - the small population size - clearly limited the power to generate a robust linkage map and to detect QTLs in avocado. Small population size exerts its primary effect by reducing the number of recombination events, leading to identical segregation of many markers, which results in their elimination as identicals in JoinMap (Van Ooijen, 2006) and a loss of marker information. 
A paucity of recombination events also results in relatively large chromosomal segments. This result, in turn, will tend to reduce the accuracy of QTL markers identified by the mapping algorithms, because the markers may be at some distance from the functional gene. Scarce recombination events may also make mapping and QTL analysis more sensitive to the stochastic nature of allelic segregation, potentially leading to the underestimation of marker distances. In outbreeding full-sib families (CP population type in JoinMap; Van Ooijen, 2006), the mapping algorithm estimates the consensus map by averaging the positions of anchor markers segregating in both parents. However, because "hk" genotypes cannot be used (in heterozygotes sharing the same two alleles, it is impossible to tell from which parent respective alleles originated), the number of informative recombination events is thus further reduced from an already small segregation pool. Segregation type will also affect QTL estimation via the IM algorithm where flanking markers are used in the calculation of LOD values for markers with uninformative segregation. While any population size will contain a proportion of markers with uninformative segregation, small populations are likely to be more heavily impacted. Because the CA-map and QTL analyses were based on the same segregating population, errors in the calculation of QTL probabilities due to a mismatch in these two components can be ruled out (Van Ooijen, 2009).

Segregation distortion (SD), a phenomenon describing loci whose alleles do not segregate according to Mendelian expectations, affects recombination between marker loci (Wang et al., 2005) and often is accused of leading to the detection of false linkage. We chose to exclude strongly SD-affected markers before generating the linkage map, though they represented $<3 \%$ of the total number of markers. This exclusion may have inadvertently removed potential QTLs, because distorted regions are as - or more-likely to contain QTLs as SD-free regions (Wang et al., 2005; Xu, 2008). In particular, SD markers are thought to be linked to loci for viability selection (Vogl and $\mathrm{Xu}, 2000$ ), including those causing inbreeding depression, a phenomenon common to outbreeding species such as avocado. While we cannot be sure that QTLs may have been missed, the loss of power arising from ignoring distorted markers is negligible in dense maps (Xu, 2008).

CANDIDATE GeNe analysis. It is disappointing that the SNPs we developed from candidate genes did not show more significant association with the nutrient phenotypes whose production the causative genes are assumed to control. One reason may be that the shortage of recombination events in our mapping population failed to detect signal. However, other factors may also be responsible. Tabor et al. (2002) argued that the candidate gene approach relies on a priori hypotheses about the role of candidate genes that may not be supported by a sufficient body of knowledge. Moreover, assumptions of gene function are generally based on studies in model organisms or major crops; yet the information may not be pertinent in avocado, an early-diverging angiosperm lineage. Further factors may be modulating effects exerted by genes outside the candidate gene pathways. Studies in Arabidopsis thaliana (Gilliland et al., 2006) and maize (Zea mays; Wang et al., 2018) identified QTLs controlling seed tocopherol content that were not part of known vitamin E pathways. In our study, CGSNPs developed to the gene encoding the enzyme homogentisate phytyl transferase (HPT1), the first committed gene in the tocopherol VTE2 biosynthetic pathway, were located in close proximity to markers significantly associated with $\alpha$-tocopherol content and were identified as significant under $\mathrm{KW}$ at both locations in 2010 but at neither location in 2009. Insufficient map resolution or uninformative segregation in the flanking markers may be responsible for the failure of IM to declare significance for the HPT1 CG-SNPs.

The only other CG-SNPs showing significant association with a phenotype (flowering type) was DXPS1, a SNP developed to a candidate gene from the vitamin B complex, that controls synthesis of a thiamine-dependent enzyme involved in cell metabolism.

Vitamin E, which consists of $\alpha$-tocopherol and several other tocopherol isomers, has been targeted by breeders pursuing crop biofortification in barley (Hordeum vulgare), maize, rapeseed (Brassica napus), rice (Oryza sativa), soybean (Glycine max), and tomato (Solanum lycopersicum) (reviewed in Fritsche et al. (2017). Peraza-Magallanes et al. (2017) found considerable variation for $\alpha$-tocopherol content in avocado germplasm from Sinaloa, Mexico. Aside from the nutritional benefits arising from elevated vitamin E levels in crops, $\alpha$-tocopherol has also been associated with enhanced tolerance of salinity and drought stress in rice and tobacco (Nicotiana tabacum) (Munné-Bosch, 2007; Ouyang et al., 2011).

Experimental populations. Avocado is a large tree that requires significant space, water, and labor resources. It takes 5 to 8 years to become productive (Lahav and Lavi, 2009), and its breeding system is very difficult to experimentally manipulate (Degani et al., 2003; Lammerts, 1942). Such cost and time considerations make it difficult and expensive to create and maintain large experimental populations and, in turn, favor working with small preexisting populations. In this regard, the UC populations used here have several strengths: 1) replication of progeny genotypes on a single clonal rootstock provides an estimate of within-genotype error variances; 2) replication in two locations provides a measure of location effects; and 3) multiple-year measurements provide a measure of temporal variance. These design features help identify important sources of environmental variance and point to important management considerations.

The current data were generated for a 'Gwen' $\times$ 'Fuerte' progeny array, and findings may not be fully transferrable to other cultivars and germplasm. However, 'Gwen'-a grandchild of 'Hass' - is central to the existing UC Riverside Breeding Program, making the QTL data relevant for MAS in the future. A crucial question to be confronted is whether QTL studies on a difficult tree crop justify the cost of land, time, and labor resources. More advanced technologies such as transformation and clustered, regularly interspaced short palindromic repeats (CRISPR)-CAS9 are appealing; but basic information about potential target genes is deficient, so for the time being MAS seems like the most practical alternative to relatively inefficient phenotypic selection. We believe that our results will encourage expanded QTL studies to guide the breeding of future cultivars in California and elsewhere, and that our findings will bring into focus the role of fruit nutritional traits with the long-term goal of breeding high-value/nutritionally enhanced cultivars achieving a market premium.

\section{Literature Cited}

Alcaraz, M.L. and J.I. Hormaza. 2009. Selection of potential pollinizers for 'Hass' avocado based on flowering time and male-female overlapping. Scientia Hort. 121:267-271. 
Ashworth, V.E.T.M., M.C. Kobayashi, M. de la Cruz, and M.T. Clegg. 2004. Microsatellite markers in avocado (Persea americana Mill.). Developing dinucleotide and trinucleotide markers. Scientia Hort. 101:255-267.

Bergh, B.O. and E. Lahav. 1996. Avocados, p. 113-166. In: J. Janick and J.N. Moore (eds.). Fruit breeding, vol. I: Tree and tropical fruits. Wiley, New York, NY.

Borrone, J.W., R.J. Schnell, H.A. Violi, and R.C. Ploetz. 2007. Seventy microsatellite markers from Persea americana Miller (avocado) expressed sequence tags. Mol. Ecol. Notes 7:439-444.

Calderón-Vázquez, C., M.L. Durbin, V.E.T.M. Ashworth, L. Tommasini, K.K.T. Meyer, and M.T. Clegg. 2013. Quantitative genetic analysis of three important nutritive traits in the fruit of avocado. J. Amer. Soc. Hort. Sci. 138:283-289.

Chen, H., V.E.T.M. Ashworth, S. Xu, and M.T. Clegg. 2007. Quantitative genetic analysis of growth rate in avocado. J. Amer. Soc. Hort. Sci. 132:691-696.

California Irrigation Management Information System. 2003. CIMIS: California Irrigation Management Information System. 17 June 2019. <https://cimis.water.ca.gov/Resources.aspx>.

D'Ambrosio, S.M. 2007. Phytonutrients: A more natural approach toward cancer prevention. Semin. Cancer Biol. 17:345-346.

Degani, C., E. Lahav, R. El-Batsri, and S. Gazit. 2003. Caging single avocado trees with a beehive does not guarantee exclusive formation of selfed progeny. HortScience 38:1433-1434.

Ding, H., C. Han, D. Guo, Y.W. Chin, Y. Ding, A.D. Kinghorn, and S.M. D'Ambrosio. 2009. Selective induction of apoptosis of human oral cancer cell lines by avocado extracts via a ROS-mediated mechanism. Nutr. Cancer 61:348-356.

Ewing, B. and P. Green. 1998. Base-calling of automated sequencer traces using phred. II. Error probabilities. Genome Res. 8:186-194.

Floral Genome Project. 2005. floralgenome.org. 17 June 2019. $<$ http:// www.floralgenome.org/>.

Fritsche, S., Z. Wang, and C. Jung. 2017. Recent advances in our understanding of tocopherol biosynthesis in plants: An overview of key genes, functions, and breeding of vitamin $\mathrm{E}$ improved crops. Antioxidants 6:99, doi: 10.3390/antiox6040099.

Gilliland, L.U., M. Magallanes-Lundback, C. Hemming, A. Supplee, M. Korneef, L. Bentsink, and D. DellaPenna. 2006. Genetic basis for natural variation in seed vitamin E levels in Arabidopsis thaliana. Proc. Natl. Acad. Sci. USA 103:18834-18841.

Gordon, D.C., C. Abajian, and P. Green. 1998. Consed: A graphical tool for sequence finishing. Genome Res. 8:195-202.

Hackett, C.A. and L.B. Broadfoot. 2003. Effects of genotyping errors, missing values and segregation distortion in molecular marker data on the construction of linkage maps. Heredity 90:33-38.

Jansen, R.C. 1993. Interval mapping of multiple quantitative trait loci. Genetics 135:205-211.

Jeong, W.S. and P.A. Lachance. 2001. Phytosterols and fatty acids in fig (Ficus carica, var. Mission) fruit and tree components. J. Food Sci. 66:278-281.

Kruglyak, L. and E.S. Lander. 1995. Nonparametric approach for mapping quantitative trait loci. Genetics 139:1421-1428.

Kruskal, W.H. and W.A. Wallis. 1952. Use of ranks in one-criterion variance analysis. J. Amer. Stat. Assn. 47:583-621.

Kuhn, D.N., D.S.I.I.I. Livingstone, J.H. Richards, P. Manosalva, N. Van den Berg, and A. Chambers. 2019. Application of genomic tools to avocado (Persea americana) breeding: SNP discovery for genotyping and germplasm characterization. Scientia Hort. 246:1-11.

Lahav, E. and U. Lavi. 2009. Avocado genetics and breeding, p. 247285. In: S.M. Jain and P.M. Priyadarshan (eds.). Breeding plantation tree crops: Tropical species. Springer-Verlag, New York, NY.

Lammerts, W.E. 1942. Progress report on avocado breeding. California Avocado Soc. Yrbk. 27:36-41.

Lander, E.S. and D. Botstein. 1989. Mapping Mendelian factors underlying quantitative traits using RFLP linkage maps. Genetics 121:185-199.

Lavi, U., E. Lahav, C. Degani, and S. Gazit. 1993. Genetics of skin colour, flowering group, and anise scent in avocado. J. Hered. 84:82-84.
Lopez-Ledesma, R., A.C. Frati-Munari, B.C. Hernandez-Dominguez, S. Cervantes-Montalvo, M.H. Hernandez-Luna, C. Juarez, and S. Moran-Lira. 1996. Monounsaturated fatty acid (avocado) rich diet for mild hypercholesterolemia. Arch. Med. Res. 27:519-523.

Luterotti, S., D. Bicanic, and R. Pozgaj. 2006. New simple spectrophotometric assay of total carotenes in margarines. Anal. Chim. Acta 573-574:466-473.

Mäeorg, E., P. Lääniste, J. Jöudu, and U. Mäeorg. 2007. Some important aspects of sterol analysis of vegetable oils. Proc. Estonian Acad. Sci. Chem. 56:59-66.

Munné-Bosch, S., E.W. Weiler, L. Alegre, M. Müller, P. Düchting, and J. Falk. 2007. $\alpha$-Tocopherol may influence cellular signaling by modulating jasmonic acid levels in plants. Planta 225:681-691.

National Center for Biotechnology Information. 2005. NCBI: National Center for Biotechnology Information. 17 June 2019. <http:// www.ncbi.nlm.nih.gov>.

Nickerson, D.A., V.O. Tobe, and S.L. Taylor. 1997. PolyPhred: Automating the detection and genotyping of single nucleotide substitutions using fluorescence-based resequencing. Nucleic Acids Res. 25:2745-2751.

Ouyang, S., S. He, P. Liu, W. Zhang, J. Zhang, and S. Chen. 2011. The role of tocopherol cyclase in salt stress tolerance of rice (Oryza sativa). Sci. China Life Sci. 54:181-188.

Peraza-Magallanes, A., M.A. Pereyra-Camacho, E. Sandoval-Castro, S. Medina-Godoy, M. Valdez-Morales, M.T. Clegg, and C.L. Calderón-Vázquez. 2017. Exploring genetic variation, oil and alpha-tocopherol content in avocado (Persea americana) from northwestern Mexico. Genet. Resources Crop Evol. 64:443-449, doi: 10.1007/s10722-016-0478-9.

R Core Team. 2019. R: A language and environment for statistical computing. 5 Apr. 2019. <http://www.R-project.org >.

Rendón-Anaya, M., E. Ibarra-Laclette, A. Méndez Bravo, T. Lan, C. Zheng, L. Carretero-Paulet, C.A. Perez-Torres, A. Chacón-López, G. Hernandez-Guzmán, T.H. Chang, K.M. Farr, W.B. Barbazuk, S. Chamala, M. Mutwil, D. Shivhare, D. Alvarez-Ponce, N. Mitter, A. Hayward, S. Fletcher, J. Rozas, A. Sánchez Gracia, D. Kuhn, A.F. Barrientos-Priego, J. Salojärvi, P. Librado, D. Sankoff, A. HerreraEstrella, V.A. Albert, and L. Herrera-Estrella. 2019. The avocado genome informs deep angiosperm phylogeny, highlights introgressive hybridization, and reveals pathogen-influenced gene space adaptation. Proc. Natl. Acad. Sci. USA, doi: 10.1073/pnas.1822129116.

Ryan, E.K., K. Galvin, T.P. O'Connor, A.R. Maguire, and N.M. O'Brien. 2007. Phytosterol, squalene, tocopherol content and fatty acid profile of selected seeds, grains, and legumes. Plant Foods Hum. Nutr. 62:85-91.

Sedgley, M. 1985. Some effects of daylength and flower manipulation on the floral cycle of two cultivars of avocado (Persea americana Mill., Lauraceae), a species showing protogynous dichogamy. J. Expt. Bot. 36:823-832.

Sedgley, M. and C.M. Annells. 1981. Flowering and fruit-set response to temperature in the avocado cultivar 'Hass'. Scientia Hort. 14:27-33.

Sharon, D., P.B. Cregan, S. Mhameed, M. Kusharska, J. Hillel, E. Lahav, and U. Lavi. 1997. An integrated genetic linkage map of avocado. Theor. Appl. Genet. 95:911-921.

Tabor, H.K., N.J. Risch, and R.M. Myers. 2002. Candidate-gene approaches for studying complex genetic traits: Practical considerations. Nat. Rev. Genet. 3:391-397, doi: 10.1038/nrg796.

The Arabidopsis Information Resource. 2005. TAIR: The Arabidopsis Information Resource. 17 June 2019. <https://www.arabidopsis.org/>. U.S. Climate Data. 2018. U.S. Climate Data. 17 June 2019. $<$ https:// www.usclimatedata.com/climate/>.

U.S. Department of Agriculture. 2018. Noncitrus fruits and nuts 2017 summary, June 2018. 17 June 2019. <http://usda.mannlib.cornell. edu/usda/current/NoncFruiNu/NoncFruiNu-06-26-2018.pdf/>.

Van Nocker, S. and S.E. Gardiner. 2014. Breeding better cultivars, faster: Applications of new technologies for the rapid deployment of superior horticultural tree crops. Hort. Res. 1:14022, doi: 10.1038/ hortres.2014.22. 
Van Ooijen, J.W. 2004. MapQTL® 5, Software for the mapping of quantitative trait loci in experimental populations. Kyazma, Wageningen, The Netherlands.

Van Ooijen, J.W. 2006. JoinMap ${ }^{\circledR}$ 4, Software for the calculation of genetic linkage maps in experimental populations. Kyazma, Wageningen, The Netherlands.

Van Ooijen, J.W. 2009. MapQTL® 6, Software for the mapping of quantitative trait loci in experimental populations of diploid species. Kyazma, Wageningen, The Netherlands.

Vogl, C. and S. Xu. 2000. Multipoint mapping of viability and segregation distorting loci using molecular markers. Genetics 155:1439-1447.
Voorrips, R.E. 2002. MapChart: Software for the graphical presentation of linkage maps and QTLs. J. Hered. 93:77-78.

Wang, C., C. Zhu, H. Zhai, and J. Wan. 2005. Mapping segregation distortion loci and quantitative trait loci for spikelet sterility in rice (Oryza sativa L.). Genet. Res. 86:97-106.

Wang, H., S. Xu, Y. Fan, N. Liu, W. Zhan, H. Liu, Y. Xiao, K. Li, Q. Pan, W. Li, M. Deng, J. Liu, M. Jin, X. Yang, J. Li, Q. Li, and J. Yan. 2018. Beyond pathways: Genetic dissection of tocopherol content in maize kernels by combining linkage and association analyses. Plant Biotechnol. J. 16:1464-1475.

$\mathrm{Xu}$, S. 2008. Quantitative trait locus mapping can benefit from segregation distortion. Genetics 180:2201-2208. 
LG 1

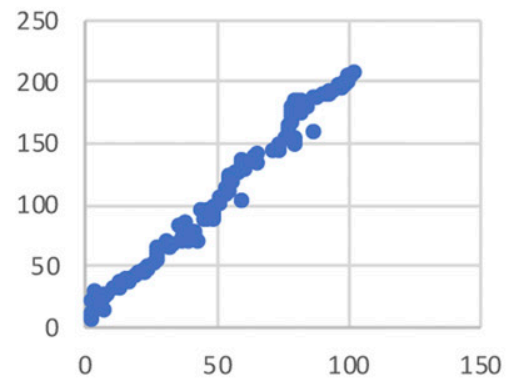

LG 4

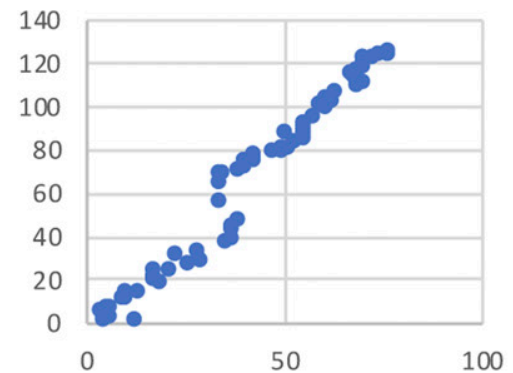

LG 7

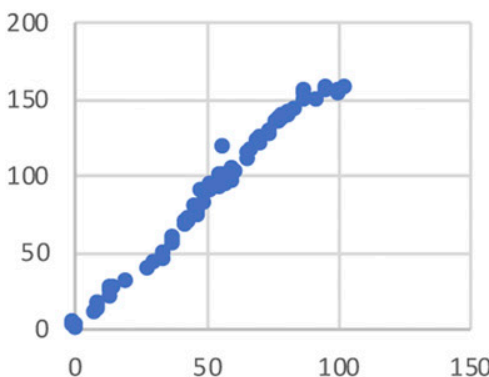

LG 10

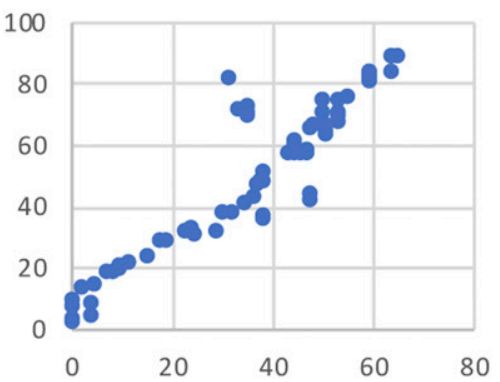

LG 2

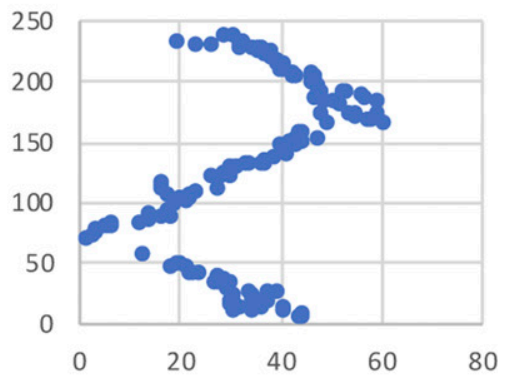

LG 5

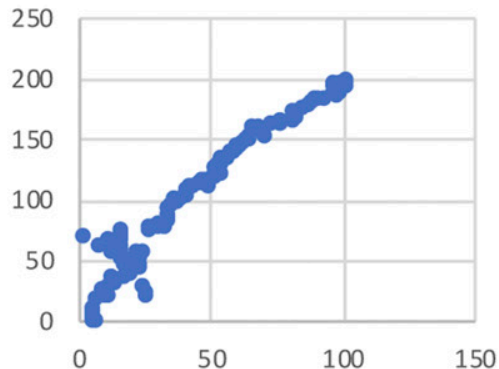

LG 8

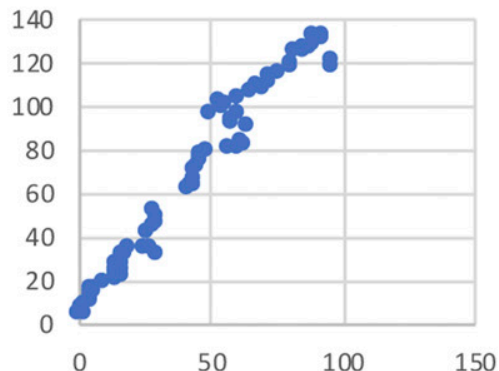

LG 11

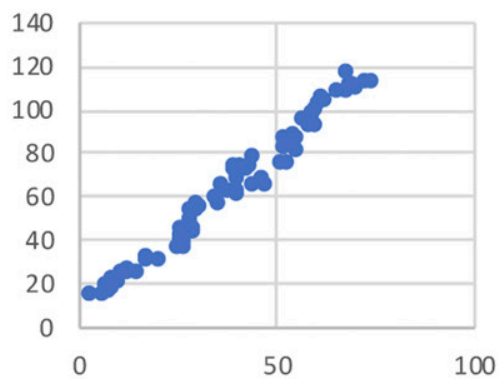

LG 3

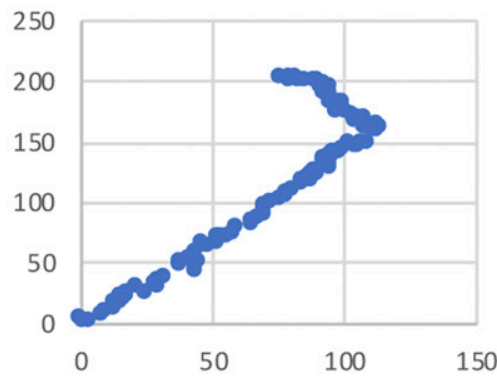

LG 6

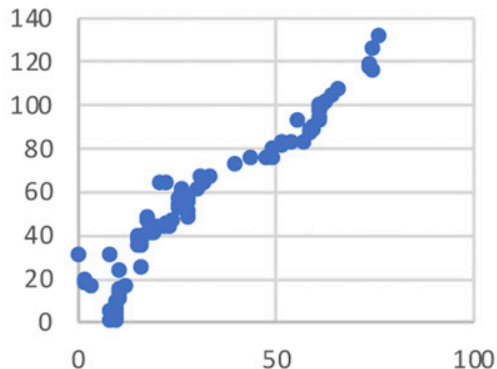

LG 9

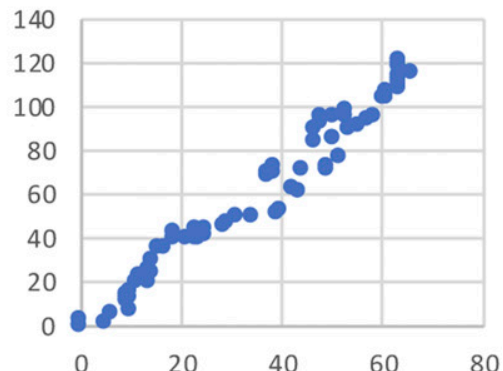

LG 12

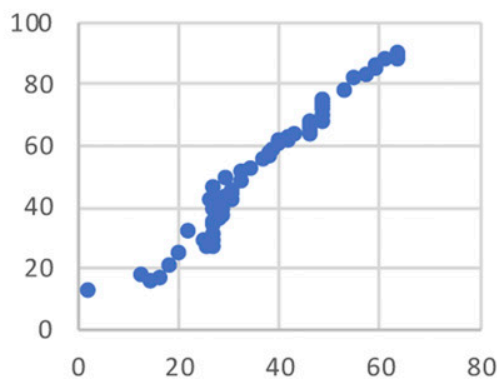

Supplemental Fig. 1. Dot plots showing map positions $[\mathrm{cM}]$ of all single nucleotide polymorphism (SNP) markers shared between the avocado 'Gwen' $\times$ 'Fuerte' California (CA)-map ( $x$ axis) (this study) and the integrated consensus linkage map of a 'Simmonds' $x$ 'Tonnage' and 'Hass' $\times$ 'Bacon' reciprocal cross [Florida (FL)-map] ( $y$ axis) (Rendón-Anaya et al., 2019). All shared markers located to the same avocado linkage group, but marker arrangements differed in some cases. 

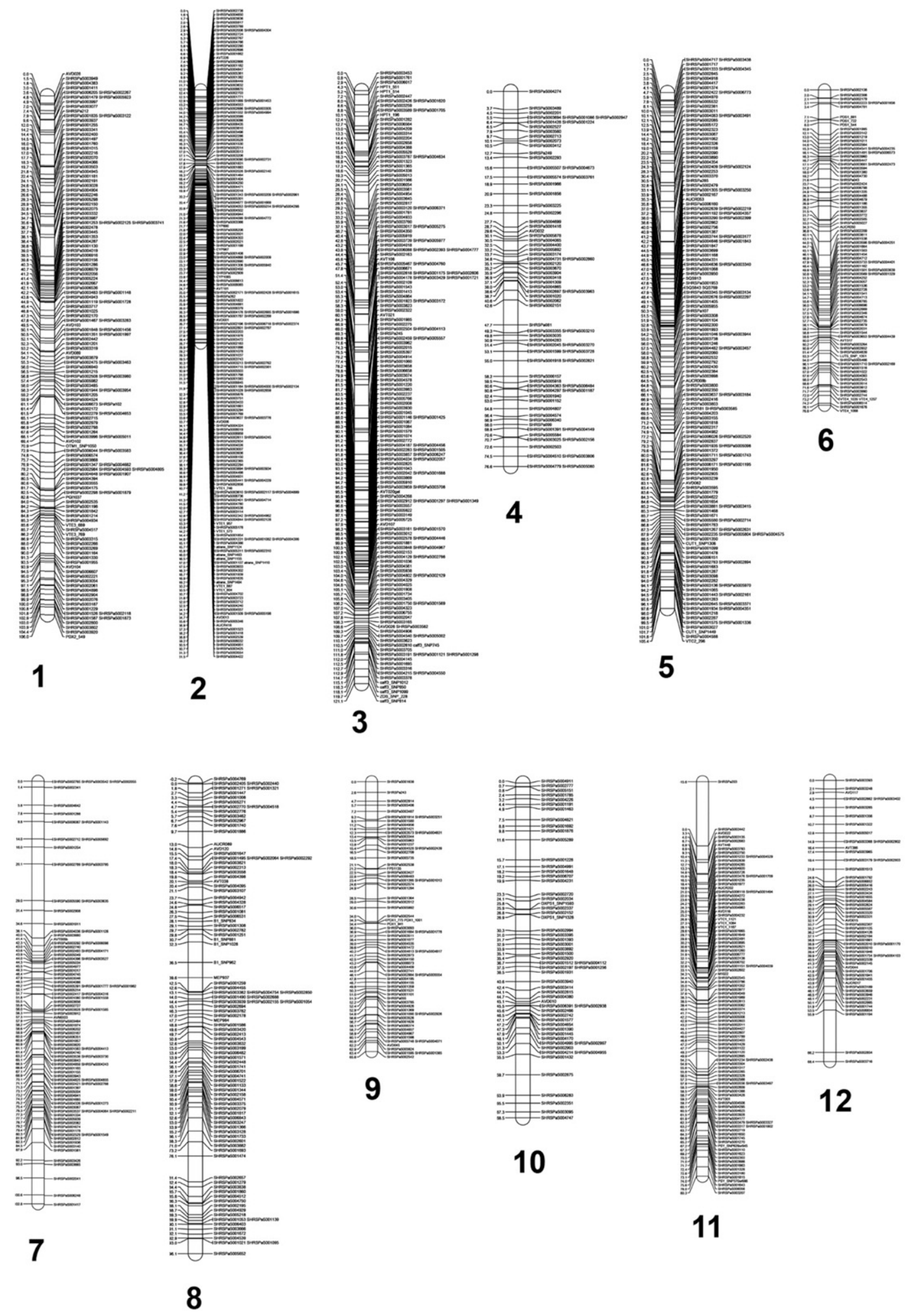

Supplemental Fig. 2. Avocado linkage map generated using JoinMap version 4 (Van Ooijen, 2006) and displayed with MapChart (Voorrips, 2002). 
Supplemental Table 1. Parameters used for single nucleotide polymorphism (SNP) discovery from candidate genes in avocado. Details are presented in the order (1) abbreviation used on the linkage map, (2) full name of enzyme encoded by the candidate gene, (3) functionally characterized gene accession found in the public databases of the National Center for Biotechnology Information (NCBI) (with the organismal source of the sequence, where given) showing the highest similarity, (4) similarity score, (5) probability of being the same gene (E-value), (6) number of SNPs detected in the gene, (7) amplifying primer (forward), (8) amplifying primer (reverse), (9) sequencing primer, annealing temperatures listed in same order as the three primers, if different.

Carotenoids

B1: Beta-carotene hydroxylase 1; At4G25700.1, 78.8², 9e-18², 1, GAA CGA TGT TTT TGC GAT CA (B1-F147), AAC AGC CCG TAT GGC ACT C (B1-R443), CGT ATG GCA CTC CAT TGA A (B1-nest509F), 64, 65, 62

LUT5: Carotene beta-ring hydroxylase, cytochrome P450-type monooxygenase; AT1G31800.1, 71.6², 1e-15², 1, ACG GTG GTA GCT CTC GTG AT (LUT-F58), TTT TTC TCT GGT TGG ATT GGA (LUT5-R473), ACG GTG GTA GCT CTC GTG AT (LUT-F58), 64, 63, 64

PSY: Phytoene synthase (PSY), geranylgeranyl-diphosphate geranylgeranyltransferase, At5G17230.1, 158², 1e-41², 2, GCT GCA TTG GCA TTA GGA AT (PSY-F22), TTG CAA TTC CTA ATG CCA (PSY-R-GAA), GGG GAT TTT ATT AGA AAA TGA (PSY-nest658R), 64, 60,56

ZDS: Zeta-carotene desaturase (ZDS), [Citrus sinensis mRNA for zeta-carotene desaturase]; emb|AJ319762.1, 89.7³ 5 e-14² 1 , TCC TCC AGA ACC TGA GCA CT (ZDS-F372), GGT TGT TGT AGC AGC CAA A (ZDS-R-GGT), CAC ATG CAG TCC CAT TTC A (ZDS-R-CAC), 64, 61,63

Darkening-related

PPO: Polyphenol oxidase (PPO); gi|311337316|gb|HQ380894.1| [Nelumbo nucifera polyphenol oxidase mRNA], 470, 1.00E-128, 1, ACC AGC TGC TTG TTC TCA TC 5093, CCC TTC CAT CGT TTC TAC CT 5094, CCC TTC CAT CGT TTC TAC CT (5094), 54

Fatty acid pathway

CUT1: Acyltransferase, Cuticular 1 (CUT 1); AT1G68530.1, 289², 2e-81², 2, CAT GGT GAT AGC TGG TGA CG, (CUT1-F27), TCT GGG ACA GAT AGG GGA TG (CUT1-R554), CATGGTGATAGCTGGTGACG (CUT1-F27), 64

Flavonoid, anthocyanin \& phenylpropanoid pathways

Caff3: Caffeoyl-CoA O-methyltransferase (caff3); Os09g30360|12009.t02714| [unspliced-genomic caffeoyl-CoA O-methyltransferase 1, putative, expressed], 91.5², 8e-22², 5, TGC GGA CAA GGA CAA CTA CA (caff3-F50), CCA TGA TGC CAT CTC TAG CA (caff3-R483), CCA AAT GGT CAA AGA AAC AG (caff3-nest1284R), 64, 64, 60

OTM1: Flavonol 3'-O-methyltransferase 1 (OTM1); gb|GU324973.1| [Eucalyptus camaldulensis caffeic O-methyltransferase1 (COMT1) gene], 66.2 $2^{3}, 8 \mathrm{e}-07^{3}, 7$, GCA GTT CTT AAG GAA TTT CGC (OTM1-F103), GGT CGA CCT ACA TAT TGC G (OTM1-R568), GAT CAC CTT TCC ATT AGC CG (OTM1-nest70F), 62, 61, 63

PAL2: Phenylalanine ammonia-lyase 2(PAL2); At3G53260.1, 702², 0.0², 2, CAG GAA TGC CAC ACT CTC AA (PAL2-F17), AGC AAA TGG GAA TAG GAG CA (PAL2-R1065), CAG GAA TGC CAC ACT CTC AA (PAL2-F17), 63-64

Isoprenoid \& sitosterol

CYP: cycloeucalenol cycloisomerase; gi|225456279|ref|XM_002283523.1| [predicted: Vitis vinifera cycloeucalenol cycloisomerase-like (LOC100262783), mRNA], 659, 0, 5, GCT TCA TAC ACC TTT CCG TCA 6163, CAT GTA GCC TCA GCA ATC CA 6162, TAG GCA TTA CGG AGT TGC AG 2130, 53

FPS: farnesyl diphosphate synthase; gi|212960745|gb|FJ415102.1| Chimonanthus praecox farnesyl pyrophosphate synthase (FPPS) mRNA, complete cds, 690, 0, 1, TTG GTT GGT TGT GAA AGC TC 634, TTG CCC AAG AAA GAC TTC AG 737, TTG GTT GGT TGT GAA AGC TC 634,53

MCR: 24-dehydrocholesterol reductase; gi|359473656|ref|XM_002271810.2| [predicted: Vitis vinifera delta(24)-sterol reductase-like (LOC100258158), mRNA], 592, 1.00E-165, 3, GGA AAG GTA TGC TTC CAA GG 20, TGT GAA GTT CAT ATA ACG AAT AGT CA 7963, TTG GCC TAG TAT CTG CAT GTT 3878, 53

SQS: squalene synthase (SQS1); gi|359475094|ref|XM_002266114.2| [predicted: Vitis vinifera squalene synthase-like (LOC100265798), mRNA], 682, 0, 4, TGA AAG TCA GTG CAT GTT TCT 6164, CGC GAC TTT GGT ATC TCA T 128, GCT TGA CCC CTT TTT TTG GA 8295,55

Vitamin B complex

atrans, Vitamin B9 (folic acid), Aminotransferase class IV family (atrans), Aminotransferase class IV family (atrans); AT5G57850.1 | Symbols: | aminotransferase class IV family protein, $66.2^{2}, 5 \mathrm{e}-14^{2}, 6$, CAG ATC CTG CAG CCA TGA TA (atrans-F-12), ACC TGT GGA GGC TTC ATT GG (atrans-R-457), TGA CAC TGC AGC TAT TAT (atrans-R457-ic-TGA), 64, 66, 51

BCAT3, Vitamin B5 (pantothenic acid), Branched-chain aminotransferase 3 (BCAT3), Branched-chain aminotransferase 3 (BCAT3); $\mathrm{gb} \mid$ EU194916.1| Nicotiana benthamiana branched-chain aminotransferase (BCAT) mRNA, $181^{3}$, 1 e-41 ${ }^{3}, 1$, CGA GGT AAA ACA TCC TAG ATC (BCAT3-F6), ACC CTT TAT TGC TGG AGT CG (BCAT3-R-ACC), GAA CCA GGA AAG CAG CAG (BCAT3-nest513F), 57, 63, 61

DXPS1, Vitamin B1 (thiamine), 1-deoxy- $D$-xylulose-5-phosphate synthase (DXPS1), 1-deoxy- $D$-xylulose-5-phosphate synthase (DXPS1); At3G21500.1 | Symbols: DXPS1 | DXPS1; 1-deoxy-D-xylulose-5-phosphate synthase, 239², 6e-66², 5, CGA GGT AAA ACA TCC TAG ATC (DXPS1-F34), AAG CAG CAG CCA AGC AGC TT (DXPS1-R-AAG), AAA TGC ATC ATA CTT TAG GAA (DXPS1-F34-R839), $57,69,55$

PDX1, Vitamin B6, Pyridoxin biosynthesis 1 (PDX1); gi|356549199|ref|XM_003542937.1| PREDICTED: Glycine max pyridoxal biosynthesis protein PDX1-like (LOC100816306), mRNA, 589, 1.00E-164, 4, CAC ACC CAA GCT GCA TCA 787, AAA TCA AGC AGG CCG TCA C 789, CAC ACC CAA GCT GCA TCA 787, 59 
Supplemental Table 1. Continued.

PDX2, Vitamin B6, Pyridoxin biosynthesis 2 (PDX2); gi|359478338|ref|XM_002285059.2| PREDICTED: Vitis vinifera pyridoxal biosynthesis protein PDX2-like (LOC100267348), mRNA, 100, 2.00E-17, 1, AAA CAG GGA AAC CTG TGT GG 779, GCC TGG TGG AAC AGC ATA AT 784, AAA CAG GGA AAC CTG TGT GG 779, 54

Vitamin C

MEP: GDP-mannose-3', 5' -epimerase, gi|359487867|ref|XM_002279341.2| PREDICTED: Vitis vinifera GDP-mannose-3' ,5' -epimerase (LOC100233034), mRNA, 437, 3.00E-119, 2, TGC TTG CAT ATA CCC AGA GTT 8889, AAG GAT TGT GTT GGC AGA CC 3058, AAG GAT TGT GTT GGC AGA CC 3058, 55

PGI: phosphoglucose isomerase, gi|225458304|ref|XM_002282738.1| PREDICTED: Vitis vinifera glucose-6-phosphate isomerase (LOC100252335), mRNA, 515, 1.00E-142, 4, TGA TAC TTG GAA AAT ACA TGA AAA CA 3881, TAA AGC CCT CAA CTG GTT CC 870, TGA TAC TTG GAA AAT ACA TGA AAA CA 3881, 54

VTC1: GDP-mannose pyrophosphorylase (VITAMIN C DEFECTIVE 1), gi|224038261|gb|FJ643600.1| Actinidia latifolia GDP-D-mannose pyrophosphorylase (GMP) mRNA, complete cds, 614, 4.00E-172, 3, GAA ACC GAG CCT CTA GGA AC 738, AGA AGC CCG GTA AGA CCA T 740, AGA AGC CCG GTA AGA CCA T 740, 56

VTC2: GDP-L galactose phosphorylase (VITAMIN C DEFECTIVE 2), gi|319739580|gb|HQ224948.1| Citrus unshiu putative GDP-L-galactosepyrophosphatase mRNA, complete cds, 246, 2.00E-61, 3, AAA ATC AAG CAT TCG CAG AG 340, CAG GCT CTT GGA GAG GTG AG 5859, AAA ATC AAG CAT TCG CAG AG 340, 55

Vitamin E

HPT1: Homogentisate phytyltransferase (VTE2), gi|219842165|dbj|AB376091.1| Hevea brasiliensis hpt mRNA for homogentisate phytyl transferase, complete cds, 347, 7.00E-92, 3, AGG CCA TTG ATA TTC GCA AC 9827, GAA ACC AAT CCC ATC ACC AC 9825, AGG CCA TTG ATA TTC GCA AC 9827, 55

PDS1: 4-hydroxyphenylpyruvate dioxygenase (PHYTOENE DESATURASE 1), gi|359485346|ref|XM_002283239.2| PREDICTED: Vitis vinifera 4-hydroxyphenylpyruvate dioxygenase-like (LOC100248785), mRNA, 558, 3.00E-155, 3, GCT GGA AAT GTG CTG ACT GA 991, TCC CAT GTC TTT TCC ATT GAC 7960, GCT GGA AAT GTG CTG ACT GA 991, 53

VTE1: Tocopherol cyclase (VITAMIN E DEFECTIVE 1, VTE1), gi|255550999|ref|XM_002516502.1| Ricinus communis Tocopherol cyclase, chloroplast precursor, putative, mRNA, 91.5, 2.00E-14, 5, GGG CAG TGC AAG AAT ATA ACT G 6564, CTC CAA GAT GGA AGT CGT GT 901, GGG CAG TGC AAG AAT ATA ACT G 6564, 53

VTE3: MPBQ/MSBQ methyltransferase (VTE3), gi|219842171|dbj|AB376094.1| Hevea brasiliensis mggbqmt mRNA for 2-methyl-6geranylgeranylbenzoquinone methyltranferase, complete cds, 814, 0, 2, TGG CTT CTT CAA TGC TCA AT 350, GCA TAA TCA GTT GGG AAT GG 5758, TGG CTT CTT CAA TGC TCA AT 350, 54

VTE4: Gamma-tocopherol methyltransferase (VTE4), gi|219842175|dbj|AB376096.1| Hevea brasiliensis gamma-tmt mRNA for gammatocopherol methyltransferase, complete cds, 381, 2.00E-102, 5, GAA CAC CAA GCC GGA AGA TA 3026, GAG AGC ACA TGC CTG ACA AA 996, GAA CAC CAA GCC GGA AGA TA 3026, 55 
Supplemental Table 2. Information on simple sequence repeat (SSR) markers of avocado, featuring marker name, source, fragment sizes in cultivars Gwen and Fuerte, distorted segregation (if applicable), forward primer, 5' to 3', reverse primer, 3' to 5', nucleotide repeat unit, annealing temperature $\left[{ }^{\circ} \mathrm{C}\right]$, and GenBank accession number.

AUCR008b, new, 268/278, 268/268, CTT CCG TAT CTC ATC AAA TA, AAA TCA GAC TCA AAT CAG TG, (CT) 22,56, KC768707 AUCR017, new, 363/370, 363/376, AAA AAG GAG TTC CAC AGT ATG A, TTC AAG TCA GAA ACC CAC TAT T, (TC) 9 (AC)9, 58, KC768708

AUCR050, new, 323/329, 329/329, GCA GAC CTG GGT TGT ATT GA, TTC GGA GCC TAT TAT TAC GAT G, (TC)18, 60, KC768709 AUCR053, new, 245/257, 245/265, AGG TTT ACA GAA GAA CCC AGA C, GAG CCC CTA CCC AAA TCT TT, (CT)6..(TC)11, 61, KC768710

AUCR089, new, 221/223, 202/221, GGC TCA TCT TCA ACT TAT, ACT CTT GTT CTT TCA GTG T, (GA)9..(GA)14, 56, KC768711 AUCR181, new, 246/246, 237/246, TTC TAT CCA GTG AGG TAA CA, CCA ATC TAT CGC CAT AAT, (GA)16, 52, KC768712 AUCR202, new, 222/222, 222/258, TGC TTA TCT TTC AAA ACC TCT G, GGC TTT ATT CTT CCC CCT AT, (GA)15, 57, KC768713 AVD010, new, 269/292, 265/265, TCT TGG AAG GTT TGG GTT TG, ATT CGG GCA GAT ACT TTC AT, (TG)5(TG)8(GA)10, 61,

$\mathrm{KC} 768714$

AVD026, new, 173/183, 173/206, AGA TAA TGA AGG TTC CAG AT, GGG AGG ATA GTA TGT AGA TTT, (AG)9, 55, KC768715 AVD028, new, 170/184, 184/184, GGG ATA TGC AAC AGA AAT ACG A, ATG GCA CGA CAA GGA AGT TC, (AG)18, 65, KC768716 AVD032, new, 179/185, 179/185, GTT TCA CCC CTT TTA ACA AGA C, AAT AGC ATA CTT GGT CTG GAG G, (GAA)2(GA)19, 63, $\mathrm{KC} 768717$

AVD036, new, 119/119, 125/125, CTT CTC CTC TTG TTC ACC CA, TAT CGG CTG TGT CTG TAT CG, (CA)3(GA)15...(CT)8..AA... (GA)12, 62, KC768718

AVD044, new, 311/313, 302/313, CTG TTG GAT GGT GTG GAT GAC, CCA GAC GTA ATG TGA GGC TCT C, (CT)15, 66, KC768719 AVD045, new, 285/285, 279/288, CCT ATG GTT TGG TGA GTT CC, TTA CAA TAC CCC TCT CGT CTG, (TTC)10(TTG)6 (GA)9, 62,

$\mathrm{KC} 768720$

AVD050, new, 186/193, 183/186, ***, CAG AAA ATC CCT AAC CCT AC, CTC TCA GAC TCG TGA CTC ATC, (GA)26, 59, KC768721 AVD065, new, 133/135, 133/135, ***, CCT TAA ACC CTC TCC CTC ATC TC, CGT GGG ATG GAT CGA AAA TG, (TC)7, 67, KC768722 AVD082, new, 113/128, 113/120, GAC CTA CTT GGA TGA GTC CT, TTG TTG TAT TGA TCT TTC CTT, (AT)5 (GT)14, 57, KC768723 AVD089, new, 256/267, 256/269, TCA TTG TGT TCT TCG TGT GGA, TAA AAG GGG TTG GTC TCA CC, (GT)13 (GA)20, 64, KC768724 AVD103, new, 181/197, 197/197, CTC CGT TCT CAT TTA TCC TC, GGT TGT CAA AAG GCT CTT AT, (CT)20, 58, KC768725 AVD104, new, 190/221, 190/221, **, TGA ACG AAA TGG AAA CAT AT, ATT TTG AAC TTT ATT GGG CT, (CG)4(TG)15(AG)22, 58, KC768726

AVD107, new, 183/191, 183/186, GCA CAC ATC AGT CGT AAA TG, TGC TAC AGG GAG AAC TTG AA, (TG)15 (AG)8, 61, KC768727 AVD116, new, 209/217, 193/217, ACA AAT GTT ATG TTT CAC CAG A, CTG TCC AAG TGT GCT AAA TG, (GA)5.C.(AG)23 , 59,

KC768728

AVD117, new, 231/231, 239/241, CGA AAG ATA GCA GGT GAG TG, GCA GTA AAG GTA GTG AAG AAT C, (GA)22, 60, KC768729 AVD120, new, 192/206, 196/206, TTC ACT ATT TTT CTT GTG GAG, AAC CAG ATG TTT CTA CAG AGA, (AG)14, 57, KC768730 AVO109, new, 152/154, 143/154, AAC TGC CTT TTC TTC TCT ATT TCA G, GGT GGG GAA CTG GGT TAG T, (TC)22, 59, KC768731 AVT001be, new, 351/365, 346/365, GGG GTA GGC AGA GGA AAT TGA A (001b.F), CCA GTC CGC ATT CAA AAG TGT T (001e.R), (TGA)8, 67, KC768732

AVT034, new, 226/228, 220/228, **, ATC GTT GTC ATC ATC GTC ATC C, CAT AGT AGG CAC TGA TGG TGT C, (TCA)5, 62, KC768733 AVT114, new, 333/345, 345/345, ***, GTT GGG ATA ATG ATT CCT GTG ATA, AGG GAA GAT GGA CCG TGA GAC C, (GAT)6(ATG)4,

$63, \mathrm{KC} 768734$

AUCR418, 2004, 359/378, 359/378, AGA TGG CTT TCT CCT TCT GA, TTT GAC ACA CAA TCC AAC TAT G, (GT)12(GA)13, 56, KC795695

AVD001, 2004, 223/238, 223/238, GTT TCC AAG CGA CTC ACG AG, GAT TCC ATG CTG AAT TGC CG, (CT)12, 66, KC795696 AVD003ii, 2004, 181/181, 184/200, TCC CTT CAG TCT AAG ATT AGC C, GAC CAA CAC TAT TTG CCC CAC, (TC)19, 62, KC795697 AVD006, 2004, 315/337, 315/298, GGG AGA GAT GTA TTG AGC A, ACT TGG TCG TAG ATT GTA AAT, (TC)9(AC)19, 56, KC795698 AVD013, 2004, 216/222, 220/243, TTG CCA GTG GAA CTT CAA AA, ACC CAA CCA AAG ATT TCA AT, (AG)7..(GA)3..(TCT)4, 62, KC795699

AVD015, 2004, 260/262, 260/260,***, GAC CCC TAC CCT AAC TCT CA, CTT CTA AAC ATT CCC TAC AAA G, (GT)26, 60, KC795700 AVD022, 2004, 226/228, 221/249, *, CCA CTT GGA TTC TTG TTG GA, ATT TGG GTT CGG CTT AGG AA, (TC)13, 65, KC795701 AVO102, 2004, 159/198, 153/169, TTC GCC TTA TCA GCG TTA G, TCT TGG AAA GCC CTA CTC C, (GA)12, 58, KC795702 AVT005b, 2004, 184/188, 184/188, TTA GCA GCA GAT AGA GGG AGA G, GGA CCT GCC TTG TGG ATT AG, (CAT)5, 62, KC795703 AVT020gat, 2004, 158/162, 158/168, CTA CAT AGA TCG AAA TAA GG, ATC TGG CTA TGA AAT GTT GG, (GAT)9, 54, KC795704 AVT021, 2004, 126/136, 126/132, *, ACT CTC GCC TCT GCG TTG AT, GAC TCA ACA TGG TTA GAA CAA GGC, (ATC)8, 65, KC795705 AVT038, 2004, 184/200, 184/184, GAT TAA AGA TGA CCC TGA AG, GAT TTG GCT CAA GAT AGA TC, (TCA)8, 56, KC795706 AVT106, 2004, 342/342, 342/336, CCA ATC AAA AGG CAA ACG AAG AAC, GCA AAG GAG GCG GTT TCG AGA T, (TCA)6, 68, KC795707

AVT158, 2004, 313/313, [313/313] ACG AAG TTA CGG GCT TAT TTC ACA, TTC TTC CCC TTC TCT CAC ATA ATC, (GAT)7, 62, KC795715 
Supplemental Table 2. Continued.

AVT191, 2004, 215/218, 215/218, TCC ACA ACT TCT ACA GGG TCG T, GGA AGA TAA CGC ACC TTG AGT TC, (ATG)7(TGG)4, 69, $\mathrm{KC} 795708$

AVT226, 2004, 298/304, 294/298, GGC TGA CTT TTA TAG TCG ATG T, TCC GAT TGA CAG TGG ATT GTT, (TCA)6..(CTT)4, 60, KC795709

AVT386, 2004, 229/229, 219/229, ACA ACC CAA ACA TAA ATG CT, AAT AGA AGT GAC ATC CGA CC, (TGA)8, 60, KC795710

AVT436, 2004, 149/152, 139/149, ***, ACT AAA ATG AGG GGA GAC TAG, GAG TGT AGT GAG GAG TTT GG, (ATC)9, 56, KC795711

AVT448, 2004, 193/193, 183/193, ACG GTG TTT GGA AGA AGA TG, GCA CTT CAA TCA ATG CTT AC, (GAT)8, 60, KC795712

AVT517, 2004, 229/229, 219/229, AAT CCT TCC ACT CAG AAA CT, TAC ACA AAC GAC AAG AAT GG, (GAT)6, 59, KC795713 AVMIX03, 2009, 145/174, 145/174, GAT ATT CCT GTT GTC ACT GC, AAT GTT CCC CAT GAA AGT CTC C, (TG)16, (AG)20, 56

SHRSPa043, 2009, 160/180, 164/180, TCA CTG CTC TCT TCT TGC CC, ATC TAT TGC CCT CTT GTA CTC ACT, (TC)2GCA(TC)14(TG) 2N6(CAAA)2, 56

SHRSPa044, 2009, 174/181, 175/175, GCC AAC GAG GGT CAG ATC AA, CGC AAA CCA ACC GCA CA, (CTT)3(TTTTAT)4, 56

SHRSPa055, 2009, 108/123, 117/137, TCT CTT CAT CAA CTC GAC TGC, AAC GGT ATC CAA ACG CTA AT, CC(TTCT)2(TTA) 2CAA(CT)16TT(CT)2, 56

SHRSPa073, 2009, 123/125, 125/125, CTG CTT TTC CCA CTG CTC, CCA GAA CAA ACT GAA CAA CAA, (AG)7AA(AG)2, 56 SHRSPa081, 2009, 218/218, 218/220, GGG CTT CAA TTC AAT CCA ATC C, TCT TCA GCA CGC CAC GAG TCT, (C)2(GA)7, 56

SHRSPa099, 2009, 79/79, 79/94, TCA TCC CAA TTC CCA CCT TC, AGC GGC GGA TTT TAG CG, (AGA)9A(AG)2, 56

SHRSPa102, 2009, 95/113, 113/119, GGC ACA AAC CCT ACA AAT ACC A, TCT TCT TGA GTC GCA GCA GC, A(GAA)6AG, 56 SHRSPa107, 2009, 151/165, 151/177, CGC AGT CTT CAA TGA TAC CA, CCC CCC TTC ACT TCC AA, (AT)4N4(AC)3TA(AC)2(CT) 2(TG)2(AGA)2AA(TG)2TAT(TC)8, 56

SHRSPa197, 2009, 164/178, 164/164, CTC TCT TCT CGA GTC CGC TG, GGA ATT CCG CAC AGT AGC AT, (CT)10CAC(CTT)3CTG(TC) 2(CTT)2, 56

SHRSPa203, 2009, 111/117, 109/111, ATG GTT ACA AGA ATT GGC CG, ATC AGT GCA AAA GGA CCC TG, (TA)2(CATA)3(TA)4, 56 SHRSPa212, 2009, 304/310, 304/304, ATT CCT TCT GCT GTC CCA AA, TGT GGC ATT AAA GAC GAC GA, (TC)5N30(CAG)2N10(GA) 2(AGAGAA)3AGA(AGC)2, 56

SHRSPa243, 2009, 260/264, 260/264, ACA GAT GAC GGT TTT CCT GC, CTC TCA GCA TCG AGC CTT TT, (ATGATTT)2CAAC(AG)8, 56

SHRSPa245, 2009, 149/151, 149/150, CCA TGA CGG AGG TTT CTT GT, GGC AAT GGC GAT TCA GTA AT, (GT)7(T)4A(AT)3(T)5(AG) 3,56

SHRSPa249, 2009, 272/276, 270/274, CCA GAA GCT GGC AAT CTA GC, CCA AAC GGG TCC TAA TGG TA, (TA)3TT(TA)9, 56 SHRSPa262, 2009, 192/195, 192/192, GGG GAA TCC ACG GCA T, TGG AGG GGA TTC TTC TCC TT, (CTT)3(CTC)4CTGCT(TCC)3, 56 SHRSPa274, 2009, 132/139, 139/139, GTG AGT CTG TAA CGC GCA GA, GCT ACA AGA TGC AGC ACC AA, (TC)21TTT(TC)2, 56 SHRSPa285, 2009, 255/264, 255/256, ACC GTT CGT TTG GAA ATC AG, GCC AAC AGT ACA TTC CCC AT, (AT)2(AGG)7(AAG)6, 56 
Supplemental Table 3. Position of genetic markers on the twelve avocado linkage groups. Quantitative trait loci (QTLs) are highlighted in bold if inferred by Interval Mapping and underlined if inferred by Kruskal-Wallis analysis. Phenotypic traits are abbreviated to A (alpha-tocopherol), B (beta-sitosterol), $\mathrm{C}$ (carotenoids), CP (canopy diameter), H (tree height), T (trunk diameter), F (flowering type).

group 1

AVD028

SHRSPaS003949

SHRSPaS004383

SHRSPaS001411

SHRSPaS006205

SHRSPaS002267

SHRSPaS001479

SHRSPaS005923

SHRSPaS003997

SHRSPaS003077

SHRSPa212

SHRSPaS001835

SHRSPaS003122

SHRSPaS003937

SHRSPaS001255

SHRSPaS003341

SHRSPaS002400

SHRSPaS001497

SHRSPaS001760

SHRSPaS001015

SHRSPaS002216

SHRSPaS002070

SHRSPaS004066

SHRSPaS003503

SHRSPaS004945

SHRSPaS001181

SHRSPaS002191

SHRSPaS003028

SHRSPaS004904

SHRSPaS002246

SHRSPaS005298

SHRSPaS002150

SHRSPaS002075

SHRSPaS003332

SHRSPaS003987

SHRSPaS001253

SHRSPaS002125

SHRSPaS003741

SHRSPaS002478

SHRSPaS003445

SHRSPaS001353

SHRSPaS004287

SHRSPaS001130

SHRSPaS004019

SHRSPaS006916

SHRSPaS003156

SHRSPaS001286

SHRSPaS006979

SHRSPaS002056

SHRSPaS005224

SHRSPaS002667
0

1.496

1.782

2.955

3.596

3.596

$4.75 \mathrm{C}$

$\underline{4.817 \mathrm{C}}$

4.879

7.016

7.442

7.905

7.905

9.59

11.981

14.167

14.425

14.46

15.963

16.878

16.981

17.657

18.294

19.677

21.771

22.436

24.148

24.637

26.288

26.591

28.294

28.862

32.832

34.45

34.69

35.563

35.592

35.592

36.73

37.667

38.098

38.391

38.614

39.033

39.476

39.839

40.345

40.706

41.216

41.435

41.923

Continued next page
Supplemental Table 3. Continued.

SHRSPaS006536

42.059

SHRSPaS003483

42.2

SHRSPaS001148

SHRSPaS004943

SHRSPaS001119

SHRSPaS001728

SHRSPaS003717

SHRSPaS001025

SHRSPaS002170

SHRSPaS001467

$\underline{\text { SHRSPaS003283 }}$

AVD103

SHRSPaS001848

SHRSPaS001456

SHRSPaS001351

SHRSPaS001997

SHRSPaS002442

SHRSPaS001201

SHRSPaS003319

AVD089

SHRSPaS003879

SHRSPaS002475

SHRSPaS003463

SHRSPaS006940

SHRSPaS001215

SHRSPaS002508

SHRSPaS003980

SHRSPaS005982

SHRSPaS003485

SHRSPaS001944

SHRSPaS003954

SHRSPaS001205

SHRSPaS004524

SHRSPaS006673

SHRSPa102

SHRSPaS002172

SHRSPaS002279

SHRSPaS004653

SHRSPaS002715

SHRSPaS002979

SHRSPaS002798

SHRSPaS001264

SHRSPaS003996

SHRSPaS005011

AVO102

OTM1_SNP1050

42.2

$43.766 \mathrm{~B}$

44.851

44.851

45.412

46.847

47.26

$47.768 \mathrm{~B}$

$47.782 \mathrm{~B}$

49.48

$50.115 \mathrm{~B}$

$50.115 \mathrm{~B}$

50.483

50.483

52.105

$52.325 \mathrm{~B}$

$53.225 \mathrm{~B}$

54.075

54.734

55.294

55.312 B

56

56.546

$56.831 \mathrm{~B}$

$\underline{56.831 \mathrm{~B}}$

57.411

58.034

$\underline{58.332 \mathrm{~B}}$

$58.332 \mathrm{~B}$

$59.238 \mathrm{~B}$

59.854

61.087 B

61.136

$61.394 \mathrm{~B}$

$61.464 \mathrm{~B}$

61.526

65.07

$65.784 \mathrm{~T}$

66.892

$67.562 \mathrm{~B}$

68.067

68.067

69.392

70.885

72.927

72.927

74.783

75.717

76.856

76.896

78.216

78.249

78.249

80.241

80.241

80.528

80.595

Continued next page 
Supplemental Table 3. Continued.

\begin{tabular}{|c|c|}
\hline SHRSPaS003 & \\
\hline SHRSPaS006670 & \\
\hline SHRSPaS002703 & \\
\hline SHRSPaS00177 & \\
\hline SHRSPaS001999 & \\
\hline SHRSPaS001453 & \\
\hline SHRSPaS00455 & \\
\hline SHRSPaS002686 & \\
\hline SHRSPaS003305 & \\
\hline SHRSPaS001552 & \\
\hline SHRSPaS00499 & \\
\hline SHRSPaS003599 & \\
\hline SHRSPaS003086 & \\
\hline SHRSPaS00643 & \\
\hline SHRSPaS003810 & \\
\hline SHRSPaS003496 & \\
\hline SHRSPaS002286 & \\
\hline SHRSPaS003528 & \\
\hline SHRSPaS001530 & \\
\hline SHRSPaS002014 & \\
\hline SHRSPaS003513 & \\
\hline SHRSPaS00320 & \\
\hline SHRSPaS003090 & \\
\hline SHRSPaS002731 & \\
\hline SHRSPaS001831 & \\
\hline SHRSPaS001883 & \\
\hline SHRSPaS002026 & \\
\hline SHRSPaS002140 & \\
\hline SHRSPaS00146 & \\
\hline SHRSPaS00140 & \\
\hline SHRSPaS004250 & \\
\hline SHRSPaS004471 & \\
\hline SHRSPaS002018 & \\
\hline SHRSPaS001343 & \\
\hline SHRSPaS00320 & \\
\hline SHRSPaS002961 & \\
\hline SHRSPaS00530 & \\
\hline SHRSPaS004677 & \\
\hline SHRSPaS00166 & \\
\hline SHRSPaS004053 & \\
\hline SHRSPaS005014 & \\
\hline SHRSPaS00429 & \\
\hline SHRSPaS004502 & \\
\hline SHRSPaS004944 & \\
\hline SHRSPaS004099 & \\
\hline SHRSPaS004772 & \\
\hline SHRSPaS003366 & \\
\hline CYP890 & \\
\hline SHRSPaS006206 & \\
\hline SHRSPaS003501 & \\
\hline SHRSPaS004303 & \\
\hline SHRSPaS00202 & \\
\hline SHRSPaS001199 & \\
\hline CYP967 & \\
\hline SHRSPaS00140 & \\
\hline SHRSPaS00486 & \\
\hline SHRSPaS00200 & \\
\hline $\begin{array}{l}\text { SHRSPaS0028 } \\
\text { SHRSPaS0013 }\end{array}$ & \\
\hline
\end{tabular}

Supplemental Table 3. Continued.

\begin{tabular}{|c|c|c|}
\hline 11.658 & SHRSPaS004175 & 81.696 \\
\hline 12.004 & SHRSPaS002298 & 82.477 \\
\hline 12.4 & SHRSPaS001879 & 82.477 \\
\hline 12.549 & PGI1037 & 82.837 \\
\hline 12.869 & $\underline{\text { SHRSPaS002535 }}$ & $84.028 \mathrm{C}$ \\
\hline 12.909 & $\overline{\text { SHRSPaS001196 }}$ & 84.155 \\
\hline 13.206 & SHRSPaS001842 & 84.331 \\
\hline 13.738 & SHRSPaS001214 & 84.751 \\
\hline 13.743 & SHRSPaS004934 & 85.315 \\
\hline 13.994 & VTE3_689 & 85.621 \\
\hline 14.025 & SHRSPaS004517 & 85.652 \\
\hline 14.239 & VTE3_769 & 86.183 \\
\hline 14.474 & SHRSPaS003315 & 86.869 \\
\hline 14.68 & SHRSPaS002266 & 88.035 \\
\hline 15.109 & $\underline{\text { SHRSPaS003269 }}$ & $89.143 \mathrm{CP}, \mathrm{T}$ \\
\hline 15.362 & $\overline{\text { SHRSPaS001164 }}$ & 89.97 \\
\hline 16.009 & SHRSPaS001330 & 91.25 \\
\hline 16.178 & SHRSPaS001955 & 93.466 \\
\hline 16.293 & AVD104 & 93.897 \\
\hline 16.946 & SHRSPaS006607 & 94.461 \\
\hline 16.959 & SHRSPaS002221 & 94.967 \\
\hline 17.12 & SHRSPaS003054 & $95.261 \mathrm{~T}$ \\
\hline 17.361 & $\overline{\text { SHRSPaS002061 }}$ & $\overline{97.05}$ \\
\hline 17.361 & SHRSPaS004896 & 98.516 \\
\hline 17.825 & SHRSPaS002904 & 98.832 \\
\hline 17.895 & SHRSPaS002076 & 99.926 \\
\hline 18.202 & SHRSPaS003187 & 99.953 \\
\hline 18.202 & SHRSPaS001229 & 100.777 \\
\hline 18.265 & SHRSPaS001526 & 101.82 \\
\hline 18.436 & SHRSPaS002118 & 101.82 \\
\hline 19.154 & SHRSPaS001587 & 102.876 \\
\hline 19.467 & SHRSPaS001873 & 102.887 \\
\hline 19.777 & SHRSPaS002800 & 103.034 \\
\hline 20.195 & SHRSPaS003802 & 103.911 \\
\hline 20.195 & SHRSPaS003920 & 104.432 \\
\hline 20.242 & PDX2_549 & 106.045 \\
\hline \multicolumn{3}{|l|}{20.242} \\
\hline 20.43 & group 2 & \\
\hline \multicolumn{3}{|l|}{20.444} \\
\hline 20.788 & SHRSPaS002738 & 0 \\
\hline 20.788 & SHRSPaS004650 & 1.641 \\
\hline 20.8 & SHRSPaS003836 & 1.688 \\
\hline 20.845 & SHRSPaS005917 & 2.044 \\
\hline 21.024 & SHRSPaS003789 & 2.593 \\
\hline 21.264 & SHRSPaS002006 & 2.943 \\
\hline 21.264 & SHRSPaS004304 & 2.943 \\
\hline 21.641 & SHRSPaS002724 & 4.251 \\
\hline 21.732 & SHRSPaS002767 & 5.282 \\
\hline 21.773 & SHRSPaS004786 & 5.743 \\
\hline 22.025 & SHRSPaS002290 & 5.809 \\
\hline 22.36 & SHRSPaS002698 & 6.055 \\
\hline 22.534 & SHRSPaS001662 & 6.638 \\
\hline 22.712 & AVT226 & 6.893 \\
\hline 22.764 & SHRSPaS002866 & 7.376 \\
\hline 22.862 & SHRSPaS001182 & 7.953 \\
\hline 22.99 & SHRSPaS004847 & 8.917 \\
\hline 23.043 & SHRSPaS005361 & 9.159 \\
\hline 23.065 & SHRSPaS001382 & 9.816 \\
\hline 23.151 & SHRSPaS006449 & 11.046 \\
\hline
\end{tabular}

11.658

12.004

12.549

12.869

13.738

14.025

14.239

14.474

14.68

15.109

15.362

16.009

16.178

16.293

16.946

17.361

17.361

17.825

17.895

19.154

19.467

19.777

20.195

20.242

20.43

20.788

20.8

21.264

21.264

21.641

21.732

21.773

22.025

22.36

22.534

22.712

22.764

22.862

22.99

23.065

23.151
SHRSPaS004175

PGI1037

SHRSPaS001214

SHRSPaS004934

VTE3_689

VTE3_769

SHRSPaS006607

PaS00222

SHRSPaS002061

SHRSPaS004896

group 2

SHRSPaS002738

SHRSPaS004650

SHRSPaS002006

SHRSPaS004304

SHRSPaS002698

SHRSPaS002866

SHRSPaS001182

SHRSPaS006449 
Supplemental Table 3. Continued.

\begin{tabular}{|c|c|c|}
\hline SHRSPaS005940 & 23.23 & SHRSPaS004245 \\
\hline SHRSPaS002450 & 23.331 & SHRSPaS00293 \\
\hline SHRSPaS002909 & 23.713 & SHRSPaS00402 \\
\hline CYP1085 & 23.849 & SHRSPaS0030C \\
\hline SHRSPaS006613 & 24.445 & SHRSPaS0039 \\
\hline SHRSPaS006065 & 24.585 & SHRSPaS0061 \\
\hline AVT191 & 24.716 & SHRSPaS0023 \\
\hline SHRSPaS002171 & 25.029 & SHRSPaS0022 \\
\hline SHRSPaS002428 & 25.029 & SHRSPaS0038 \\
\hline SHRSPaS001615 & 25.03 & SHRSPaS0039 \\
\hline SHRSPa262 & 25.487 & SHRSPaS0044 \\
\hline SHRSPaS001822 & 25.571 & SHRSPaS0049 \\
\hline SHRSPaS004881 & 25.85 & SHRSPaS0054 \\
\hline SHRSPaS006379 & 25.999 & SHRSPaS0042 \\
\hline SHRSPaS004176 & 26.256 & $\underline{\text { SHRSPaS0028 }}$ \\
\hline SHRSPaS002685 & 26.314 & VTE1_746 \\
\hline SHRSPaS001686 & 26.339 & SHRSPaS0036 \\
\hline SHRSPaS001787 & 26.467 & SHRSPaS0021 \\
\hline SHRSPaS002269 & 26.504 & SHRSPaS0048 \\
\hline AVD001 & 26.596 & SHRSPaS00673 \\
\hline SHRSPaS002196 & 26.671 & SHRSPaS0024 \\
\hline SHRSPaS006718 & 26.698 & SHRSPaS0047 \\
\hline SHRSPaS002374 & 26.723 & SHRSPaS0047 \\
\hline SHRSPaS002801 & 27.087 & SHRSPaS0045 \\
\hline SHRSPaS002787 & 27.087 & SHRSPaS0031 \\
\hline SHRSPaS002203 & 27.509 & SHRSPaS0023 \\
\hline SHRSPaS001976 & 27.606 & SHRSPaS0049 \\
\hline SHRSPaS003472 & 28.419 & SHRSPaS0048 \\
\hline SHRSPaS002183 & 28.704 & SHRSPaS00212 \\
\hline SHRSPaS001998 & 28.909 & VTE1_957 \\
\hline SHRSPaS001078 & 29.182 & SHRSPaS0051 \\
\hline SHRSPaS001037 & 29.276 & VTE1_573 \\
\hline SHRSPaS003743 & 29.493 & SHRSPaS0018 \\
\hline SHRSPaS003213 & 30.268 & SHRSPaS0012 \\
\hline SHRSPaS002762 & 30.349 & SHRSPaS0010 \\
\hline SHRSPaS004715 & 30.521 & SHRSPaS00430 \\
\hline SHRSPaS002561 & 30.521 & SHRSPaS0043 \\
\hline SHRSPaS005503 & 30.929 & atrans_SNP1124 \\
\hline SHRSPaS001768 & 30.995 & SHRSPaS0053 \\
\hline SHRSPaS001593 & 31.305 & SHRSPaS0023 \\
\hline SHRSPaS006845 & 31.542 & atrans_SNP149. \\
\hline SHRSPaS001184 & 31.853 & atrans_SNP1155 \\
\hline SHRSPaS004000 & 31.894 & SHRSPaS0011? \\
\hline SHRSPaS002134 & 31.894 & atrans_SNP1410 \\
\hline SHRSPaS001029 & 31.913 & SHRSPaS0039 \\
\hline SHRSPaS002659 & 31.945 & SHRSPaS0043 \\
\hline SHRSPaS005876 & 32.289 & SHRSPaS0019 \\
\hline SHRSPaS003751 & 32.412 & $\underline{\text { SHRSPaS0016 }}$ \\
\hline SHRSPaS003433 & 32.822 & atrans_SNP1484 \\
\hline SHRSPaS001661 & 32.908 & VTE1_687 \\
\hline SHRSPaS003294 & 33.231 & VTE1_604 \\
\hline SHRSPaS001769 & 33.478 & $\underline{\text { SHRSPaS0047 }}$ \\
\hline SHRSPaS003627 & 33.583 & SHRSPaS0037 \\
\hline SHRSPaS003776 & 33.601 & SHRSPaS0037 \\
\hline AVD006 & 33.666 & SHRSPaS0042 \\
\hline SHRSPaS004324 & 34.073 & SHRSPaS0045 \\
\hline SHRSPaS006019 & 34.319 & $\underline{\text { SHRSPaS0015 }}$ \\
\hline SHRSPaS006959 & 34.35 & SHRSPaS0051 \\
\hline SHRSPaS002611 & 34.624 & AVD013 \\
\hline
\end{tabular}

Continued next page
34.624

34.757

35.673

36.306

36.414

37.555

37.688

38.139

38.263

38.263

38.641

38.934

39.106

39.106

$\underline{39.765 \mathrm{~A}}$

40.708

41.156

41.16

41.16

41.207

41.263

41.32

41.505

41.987

42.671

42.766

42.846

42.916

42.929

43.284

43.763

44.23

44.449

44.799

44.799

44.839

45.052

45.685

45.828

45.828

45.955

46.47

46.962

47.013

47.457

48.301

48.704

$48.947 \mathrm{~A}$

$49.205 \mathrm{~T}$

50.245

50.473

$50.683 \mathrm{~A}$

51.783

52.034

52.485

54.085

$54.156 \mathrm{~A}$

54.219

54.651

Continued next page 
Supplemental Table 3. Continued.

SHRSPaS005346
AUCR418
SHRSPaS001523
SHRSPaS001418
SHRSPaS002539
SHRSPaS006032
SHRSPaS004951
SHRSPaS003425
SHRSPaS003554
SHRSPaS004422

group 3

$\underline{\text { SHRSPaS003453 }}$ SHRSPaS001761 SHRSPaS006017

HPT1_551

HPT1 514

SHRSPaS002447

SHRSPaS002426

SHRSPaS001620

SHRSPaS003259

SHRSPaS003589

SHRSPaS001705 HPT1_196

SHRSPaS001282

SHRSPaS006564

SHRSPaS004209

SHRSPaS003314

SHRSPaS002204

SHRSPaS002658

SHRSPaS004388

SHRSPaS005529

SHRSPaS003787

SHRSPaS004634

SHRSPaS001323

SHRSPaS001365

SHRSPaS004338

SHRSPaS005013

SHRSPaS001566

SHRSPaS006054

SHRSPaS003561

SHRSPaS004954

SHRSPaS003645

SHRSPaS002817

SHRSPaS003120

SHRSPaS006371

SHRSPaS001781

SHRSPaS004933

SHRSPaS003760

SHRSPaS003017

SHRSPaS005275

SHRSPaS004350

SHRSPaS005919

SHRSPaS003739

SHRSPaS005977

SHRSPaS004018

SHRSPaS006088

SHRSPaS002393
55.582

56.929

57.267

58.203

58.75

59.165

60.469

60.742

61.27

61.483

$\underline{0 \mathrm{~A}}$

0.82

2.938

$4.297 \mathrm{~A}$

$\overline{5.196 \mathrm{~A}}$

$7.242 \mathrm{~A}$

$7.968 \mathrm{~A}$

$\mathbf{7 . 9 6 8 \mathrm { A }}$

$8.562 \mathrm{~A}$

$8.847 \mathrm{~A}$

$8.847 \mathrm{~A}$

$10.078 \mathrm{~A}$

$12.359 \mathrm{~A}$

$12.736 \mathrm{~A}$

$12.998 \mathrm{~A}$

13.349 A

$14.43 \mathrm{~A}$

$14.63 \mathrm{~A}$

$15.363 \mathrm{~A}$

15.648 A

16.304 A

16.304 A

$16.785 \mathrm{~A}$

17.332 A

18.601 A

19.334

$20.682 \mathrm{~A}$

$24.144 \mathrm{~B}$

$25.5 \mathrm{~A}$

26.428

$27.638 \mathrm{~A}$

28.082

29.223

29.223

31.467

33.176

35.949

37.112

37.112

38.714

40.402

42.778

42.778

43.234

43.779

43.793
Supplemental Table 3. Continued.

SHRSPaS004777

43.793

SHRSPaS002163

44.44

AVT106

45.573

SHRSPaS005467 $\quad 45.724$

SHRSPaS004760 $\quad 45.724$

SHRSPaS006671 $\quad 47.806$

SHRSPaS002618 $\quad 51.357$

SHRSPaS001175 $\quad 51.357$

SHRSPaS002606 51.384

SHRSPaS004178 $\quad 51.384$

SHRSPaS003429 51.438

SHRSPaS001721 51.438

SHRSPaS002109 $\quad 52.417$

SHRSPaS001543 $\quad 53.001$

SHRSPaS001413 53.351

SHRSPaS004864 55.314

SHRSPaS001923 $\quad 57.692$

SHRSPaS003172 57.692

SHRSPaS003823 $\quad 58.283$

SHRSPaS002322 58.956

AVT021

SHRSPaS001985

SHRSPaS002275

SHRSPaS002504

SHRSPaS004113

SHRSPa245

SHRSPaS002459

SHRSPaS005557

SHRSPaS003962

SHRSPaS002015

SHRSPaS005397

SHRSPaS004914

SHRSPaS001060

SHRSPaS003658

SHRSPaS006658

SHRSPaS003074

SHRSPaS004578

SHRSPaS001220

SHRSPaS003983

SHRSPaS002237

SHRSPaS005766

60.065

64.309

66.882

68.988

68.988

69.281

69.755

69.755

71.614

74.346

75.484

76.205

77.405

78.24

78.799

79.865

80.978

81.536

81.681

83.475

84.518

84.829

85.979

87.354

87.997

87.997

88.099

89.34

90.134

90.363

91.171

91.398

91.398

91.553

91.639

91.76

91.76

92.393

92.434

SHRSPaS004034

SHRSPaS002057 
Supplemental Table 3. Continued.

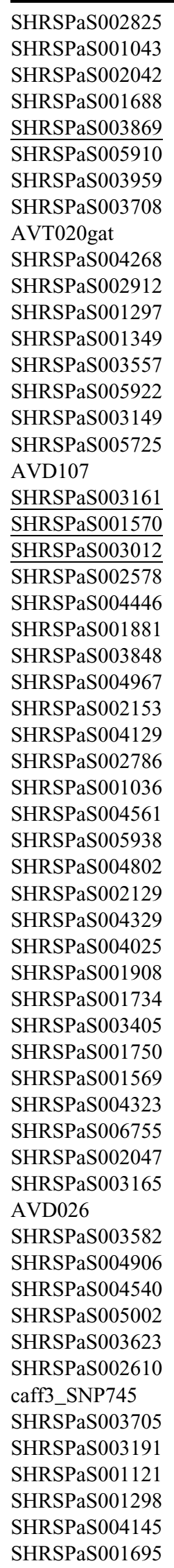

Supplemental Table 3. Continued.

\begin{tabular}{ll}
\hline SHRSPaS003316 & 112.658 \\
SHRSPaS004215 & 112.867 \\
SHRSPaS004550 & 112.905 \\
SHRSPaS003378 & 114.667 \\
caff3_SNP1012 & 115.113 \\
caff3_SNP850 & 116.291 \\
caff3_SNP1099 & 118.125 \\
ZDS_SNP_228 & 119.741 \\
caff3_SNP814 & 121.125
\end{tabular}

group 4

SHRSPaS004274 0

SHRSPaS003489 3.71

SHRSPaS002201 $\quad 4.519$

SHRSPaS003694 5.476

SHRSPaS001086 $\quad 5.505$

SHRSPaS002947 $\quad 5.505$

SHRSPaS001428 6.431

SHRSPaS001224 6.431

SHRSPaS002527 $\quad 6.487$

SHRSPaS003560 $\quad 7.928$

SHRSPaS002713 $\quad 9.719$

SHRSPaS002073 $\quad 10.118$

SHRSPaS003412 10.492

SHRSPa249 12.654

SHRSPaS002293 13.388

SHRSPaS005507 $\quad 15.622$

SHRSPaS004673 $\quad 15.622$

SHRSPaS005574 $\quad 17.487$

SHRSPaS003761 17.487

SHRSPaS001966 18.916

SHRSPaS001856 20.868

SHRSPaS003225 23.349

SHRSPaS002296 24.762

SHRSPaS004699 27.68

SHRSPaS001416 28.68

AVD032 29.556

SHRSPaS005878 30.148

SHRSPaS004065 30.5

SHRSPaS004400 32.508

SHRSPaS005892 $\quad 33.172$

SHRSPaS003174 33.727

SHRSPaS004731 34.796

SHRSPaS002860 34.796

SHRSPaS002120 35.133

SHRSPaS003670 $\quad 35.165$

SHRSPaS003904 35.947

SHRSPaS003418 36.926

SHRSPaS001309 37.139

SHRSPaS004865 38.006

SHRSPaS002697 38.573

SHRSPaS003963 38.573

SHRSPaS001020 38.656

SHRSPaS002062 40.595

SHRSPaS002151 $\quad 42.569$

SHRSPa081

SHRSPaS003355

SHRSPaS003210

$47.675 \mathrm{~A}$

49.264

49.264

Continued next page 
Supplemental Table 3. Continued.

\begin{tabular}{l}
\hline SHRSPaS003035 \\
SHRSPaS004283 \\
\hline SHRSPaS002045 \\
SHRSPaS003270 \\
SHRSPaS001599 \\
SHRSPaS003728 \\
SHRSPaS001918 \\
SHRSPaS002821 \\
SHRSPaS006157 \\
SHRSPaS005918 \\
SHRSPaS004363 \\
SHRSPaS006484 \\
SHRSPaS004297 \\
SHRSPaS001187 \\
SHRSPaS001940 \\
SHRSPaS001152 \\
SHRSPaS004807 \\
SHRSPaS004574 \\
\hline SHRSPaS006340 \\
SHRSPa099 \\
\hline SHRSPaS001391 \\
\hline SHRSPaS004149 \\
\hline SHRSPaS005584 \\
SHRSPaS003025 \\
\hline SHRSPaS002156 \\
\hline SHRSPaS002503 \\
SHRSPaS004510 \\
SHRSPaS003806 \\
SHRSPaS004779 \\
SHRSPaS005080
\end{tabular}

group 5

SHRSPaS004717

SHRSPaS003438

SHRSPaS001717

SHRSPaS001333

SHRSPaS004345

SHRSPaS002845

SHRSPaS004918

SHRSPaS004417

SHRSPaS001374

SHRSPaS002422

SHRSPaS006773

SHRSPaS004970

SHRSPaS005532

SHRSPaS002381

SHRSPaS003011

SHRSPaS004083

SHRSPaS003491

SHRSPaS002085

SHRSPaS005172

SHRSPaS002323

SHRSPaS003067

SHRSPaS001062

SHRSPaS002326

SHRSPaS003159

SHRSPaS002090

SHRSPaS003890
49.799

50.889A

51.427

51.427

53.104

53.104

55.033

55.033

58.152

59.503

60.623

60.623

60.846

60.846

62.417

63.042

64.759

$\underline{66.427 \mathrm{~A}}$

66.904

$\underline{67.989 \mathrm{~A}}$

$68.806 \mathrm{~A}$

$68.806 \mathrm{~A}$

70.569

$70.718 \mathrm{~A}$

$\overline{70.718 \mathrm{~A}}$

72.624

74.513

74.513

76.59

76.59

0

0

1.497

1.729

1.729

2.542

3.044

3.837

4.084

4.696

4.696

5.929

7.941

8.074

10.028

10.079

10.079

12.132

12.461

13.873

14.41

16.227

18.388

19.027

20.155

22.174
Supplemental Table 3. Continued.

SHRSPaS004354

SHRSPaS002409

SHRSPaS002124

SHRSPaS002253

SHRSPaS003370

SHRSPa285

SHRSPaS002479

SHRSPaS001305

SHRSPaS003250

SHRSPaS002167

AUCR053

SHRSPaS006160

SHRSPaS002639

SHRSPaS002219

SHRSPaS001192

SHRSPaS004357

SHRSPaS003290

SHRSPaS002399

SHRSPaS002862

SHRSPaS002756

SHRSPaS001387

SHRSPaS003744

SHRSPaS003177

SHRSPaS004646

SHRSPaS001843

SHRSPaS001847

SHRSPaS003699

SHRSPaS001168

SHRSPaS004331

SHRSPaS004636

SHRSPaS003340

SHRSPaS001068

SHRSPaS003950

SQS913

SHRSPaS001953

SQS843

SQS769

SHRSPaS003345

SHRSPaS003134

SHRSPaS002676

SHRSPaS002297

SHRSPaS001405

SHRSPaS005955

SHRSPa107

SHRSPaS003308

SHRSPaS001104

SHRSPaS002300

SHRSPaS001993

SHRSPaS001046

SHRSPaS003944

SHRSPaS003738

SHRSPaS001246

SHRSPaS004482

SHRSPaS003457

SHRSPaS002060

SHRSPaS002532

SHRSPaS002792

SHRSPaS002430

SHRSPaS002384
23.46

24.309

24.309

28.261

30.437

30.495

32.822

33.132

33.132

33.944

35.318

35.832

36.965

36.965

37.198

37.198

37.962

37.962

38.557

39.973

40.935

41.2

41.2

42.222

42.222

43.704

44.482

44.892

45.536

46.567

46.567

46.994

47.079

47.547

47.574

47.689

47.689

47.886

47.886

48.351

48.351

49.074

49.712

50.306

51.546

51.922

52.798

53.855

54.152

54.152

55.035

55.99

57.458

57.458

58

58.814

59.919

60.217

62.505

Continued next page 
Supplemental Table 3. Continued.

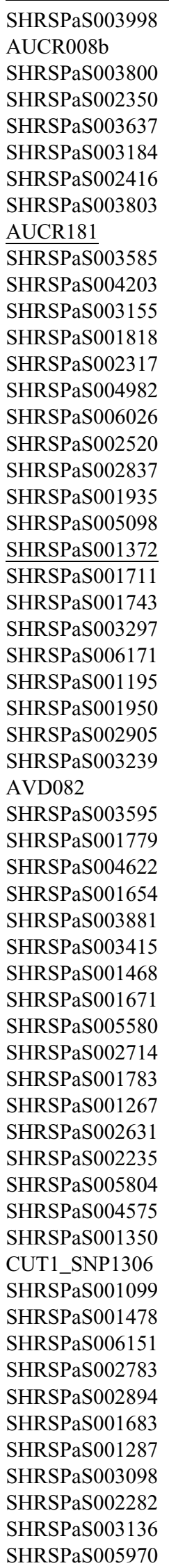

Supplemental Table 3. Continued.

SHRSPaS001065

SHRSPaS001443

SHRSPaS002161

SHRSPaS001283

SHRSPaS002645

SHRSPaS003371

SHRSPaS001934

SHRSPaS004351

SHRSPaS001218

SHRSPaS002287

SHRSPaS001575

SHRSPaS001336

SHRSPaS003027

CUT1_SNP1449

SHRSPaS004588

VTC2_296

group 6

79.158

79.307

79.307

$79.552 \mathrm{~B}$

79.961

79.995

80.872

81.644

81.644

81.685

82.637

82.796

83.12

83.44

83.561

84.326

84.624

85.174

85.174

85.277

85.662

86.313

86.313

86.831

87.253

87.314

87.937

87.937

87.942

88.51

89.121

89.578

90.057

90.292

90.798

90.839

91.68

91.805

92.094

94.01

94.101

94.101

\section{SHRSPaS002138}

SHRSPaS002308

SHRSPaS002179

SHRSPaS002223

SHRSPaS001656

SHRSPaS004781

PDS1_881

PDS1_722

PDS1_544

SHRSPaS001995

SHRSPaS003142

SHRSPaS001219

SHRSPaS001011

SHRSPaS002564

SHRSPaS004541

SHRSPaS004235

SHRSPaS001491

SHRSPaS006573

SHRSPaS003960

SHRSPaS005447

SHRSPaS003681

SHRSPaS002473

SHRSPaS003594

SHRSPaS001380

SHRSPaS004730

SHRSPa043

SHRSPaS002424

SHRSPaS006788

SHRSPaS001335

SHRSPaS004488

SHRSPaS004679

SHRSPaS001186

SHRSPaS001355

SHRSPaS003837

SHRSPaS003772

SHRSPaS002505

SHRSPaS005027

AUCR050

SHRSPaS002209

SHRSPaS003811
94.201

94.479

94.479

95.518

96.052

96.052

97.576

97.599

98.013

98.911

99.526

99.526

100.976

101.69

101.755

105.432

0

2.036

2.057

3.124

3.124

$\underline{3.413 \mathrm{C}}$

7.053

7.269

7.967

10.87

12.145

12.955

14.029

14.586

15.257

15.332

16.133

16.133

16.273

16.671

17.077

17.077

17.2

18.04

19.216

21.618

23.714

24.824

24.868

26.295

26.831

26.944

28.248

31.527

31.717

32.661

33.196

$\underline{34.272 \mathrm{C}}$

36.754

$40.129 \mathrm{~F}$

Continued next page 
Supplemental Table 3. Continued.

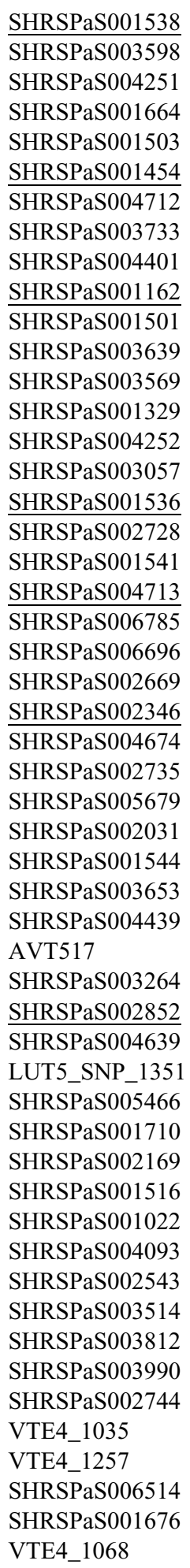

group 7

SHRSPaS002765

SHRSPaS003542

SHRSPaS002055

SHRSPaS002341

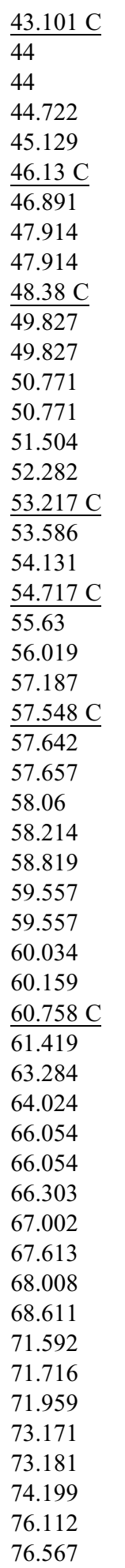

0
0
0
1.43

Continued next page
Supplemental Table 3. Continued.

\begin{tabular}{ll}
\hline SHRSPaS004642 & 5.77 \\
SHRSPaS001288 & 7.81 \\
SHRSPaS006087 & 9.83 \\
SHRSPaS001143 & 9.835
\end{tabular}

SHRSPaS002712 13.951

SHRSPaS005692 13.951

SHRSPaS001254 15.95

SHRSPaS002789 20.106

SHRSPaS003795 20.106

SHRSPaS005590 28.967

SHRSPaS003635 28.967

SHRSPaS002908 31.364

SHRSPaS001911 $\quad 34.608$

SHRSPaS004036 $\quad 38.092$

SHRSPaS001128 $\quad 38.092$

SHRSPaS003660 40.049

AVT005b $\quad 41.438$

SHRSPaS003292 $\quad 42.302$

SHRSPaS006098 $\quad 42.302$

SHRSPaS002740 $\quad 42.95$

SHRSPaS002493 43.577

SHRSPaS004171 43.577

SHRSPaS005049 $\quad 43.877$

SHRSPaS004396 44.014

SHRSPaS003527 44.014

SHRSPaS005034

SHRSPaS004977

SHRSPaS001017

SHRSPaS004745

SHRSPaS003046

SHRSPaS004086

SHRSPaS005391

SHRSPaS001777

SHRSPaS001982

SHRSPaS005314

SHRSPaS002417

SHRSPaS004316

$44.338 \mathrm{CP}$

45.476

46.308

46.374

47.916

48.296

49.181

49.234

49.234

50.066

51.661

51.661

51.792

51.829

55.462

55.753

55.993

55.993

56.342

57.273

$57.902 \mathrm{~A}$

58.197

58.641

58.916

59.97

60.769

60.924

61.076

61.076

61.383

63.158

63.158

65.118

66.458

SHRSPaS003843

SHRSPaS004859

Continued next page 
Supplemental Table 3. Continued.

SHRSPaS004243
SHRSPaS001165
SHRSPaS001155
SHRSPaS003943
SHRSPaS004825
SHRSPaS004855
SHRSPaS002421
SHRSPaS002768
SHRSPaS001397
SHRSPaS005004
SHRSPaS004941
SHRSPaS001685
SHRSPaS004326
SHRSPaS001273
SHRSPaS003087
SHRSPaS003537
SHRSPaS004064
SHRSPaS002211
SHRSPaS001334
SHRSPaS005939
SHRSPaS002082
SHRSPaS001674
SHRSPaS001178
SHRSPaS002529
SHRSPaS001549
SHRSPaS002812
SHRSPaS001936
SHRSPaS003140
SHRSPaS001561
SHRSPaS003426
SHRSPaS003665
SHRSPaS002041
SHRSPaS006248 001417

group 8

SHRSPaS004769
SHRSPaS002405
SHRSPaS002440
SHRSPaS001271
SHRSPaS001321
SHRSPaS001447
SHRSPaS001008
SHRSPaS005271
SHRSPaS002770
SHRSPaS004518
SHRSPaS002776
SHRSPaS003462
SHRSPaS002967
SHRSPaS001740
SHRSPaS001886
AUCR089
AVD120
SHRSPaS001647
SHRSPaS001495
SHRSPaS002064
SHRSPaS002292
SHRSPaS003821

66.458

66.64

67.112

68.947

70.072

70.072

71.113

71.113

71.324

73.063

73.206

74.199

75.207

75.207

75.407

76.283

76.283

76.323

77.288

77.71

78.718

79.237

79.383

80.322

80.322

81.914

82.25

84.516

87.923

92.232

93.013

96.47

100.563

102.78

$-0.213$

0

0

1.8

1.8

2.706

3.328

4.385

4.741

4.741

5.367

5.678

6.711

7.579

9.702

12.983

14.763

15.543

17.419

17.432

17.436

17.958

Supplemental Table 3. Continued.

18.26

SHRSPaS003558

18.412

SHRSPaS004398

19.77

AVT038

20.061

SHRSPaS004095

20.379

SHRSPaS003107

21.122

SHRSPaS004942

23.729

24.552

SHRSPaS006517 24.818

$\begin{array}{ll}\text { SHRSPaS001081 } & 26.287\end{array}$

SHRSPaS006531

B1_SNP834

SHRSPaS001308

27.54

28.094

29.08

29.727

29.771

SHRSPaS001251

30.736

B1_SNP881

B1_SNP1028

B1_SNP962

32.272

MEP937

SHRSPaS001259

36.536

39.587

42.546

42.557

43.082

43.098

43.098

43.958

43.958

44.416

44.416

44.428

45.138

46.3

46.998

47.706

48.833

49.552

49.683

50.838

52.642

53.02

53.441

53.859

54.435

56.094

56.834

57.098

57.935

58.62

59.001

59.554

60.417

60.885

61.085

62.094

62.606

63.012

63.921

65.152

66.105

68.303

Continued next page 
Supplemental Table 3. Continued.

\begin{tabular}{l}
\hline SHRSPaS003882 \\
SHRSPaS001693 \\
SHRSPaS001474 \\
SHRSPaS002657 \\
SHRSPaS001279 \\
SHRSPaS003838 \\
SHRSPaS001860 \\
SHRSPaS004512 \\
SHRSPaS004750 \\
\hline SHRSPaS002195 \\
\hline SHRSPaS004929 \\
\hline SHRSPaS005218 \\
SHRSPaS001053 \\
SHRSPaS001139 \\
SHRSPaS006403 \\
SHRSPaS003666 \\
SHRSPaS001672 \\
SHRSPaS004539 \\
SHRSPaS001021 \\
SHRSPaS001095 \\
SHRSPaS005652
\end{tabular}

group 9

SHRSPaS001638

SHRSPa243

SHRSPaS002814

SHRSPaS005406

SHRSPaS003487

SHRSPaS001914

SHRSPaS003251

SHRSPaS001580

SHRSPaS004956

SHRSPaS001421

SHRSPaS003573

SHRSPaS004831

SHRSPaS003344

SHRSPaS005963

SHRSPaS001237

SHRSPaS004520

SHRSPaS002439

SHRSPaS002709

SHRSPaS005735

SHRSPaS002538

FPS1135

SHRSPaS003427

SHRSPaS006483

SHRSPaS001395

SHRSPaS001013

SHRSPaS002574

SHRSPaS001284

SHRSPaS001364

SHRSPaS002012

SHRSPaS005992

SHRSPaS002544

PDX1_775

PDX1_1001

PDX1_941

SHRSPaS003093
71.02

73.188

76.122

81.443

82.43

84.438

85.722

85.761

86.3 A

$88.125 \mathrm{~A}$

$\underline{88.655 \mathrm{~A}}$

89.329

89.871

89.871

90.057

91.135

92.098

92.928

92.986

92.986

96.073

0

2.563

4.658

5.705

7.177

9.174

9.174

9.501

11.214

11.636

12.287

12.287

13.353

13.487

13.826

15.419

15.419

16.501

18.52

21.062

21.199

22.533

22.753

23.351

23.351

24.633

24.888

28.495

28.996

30.586

33.984

34.29

34.316

34.412

36.045
Supplemental Table 3. Continued.

SHRSPaS001684

SHRSPaS001778

SHRSPaS003511

SHRSPaS001077

SHRSPaS004535

SHRSPaS001472

SHRSPaS004613

SHRSPaS004817

SHRSPaS002973

SHRSPaS004150

SHRSPaS006073

SHRSPaS004409

SHRSPaS002741

SHRSPaS002884

SHRSPaS005554

SHRSPaS004195

SHRSPaS004155

SHRSPaS001359

SHRSPaS001531

SHRSPaS001101

SHRSPa055

SHRSPaS003785

SHRSPaS004926

SHRSPaS001369

SHRSPaS001090

SHRSPaS002926

SHRSPaS003526

SHRSPaS001628

SHRSPaS006374

SHRSPaS004657

SHRSPaS004867

SHRSPaS001598

SHRSPaS005746

SHRSPaS004071

AVD045

SHRSPaS005924

SHRSPaS001595

SHRSPaS001385

SHRSPaS002542

group 10

SHRSPaS004911

SHRSPaS002777

SHRSPaS005151

SHRSPaS001785

SHRSPaS004226

SHRSPaS001191

SHRSPaS001463

SHRSPaS004821

SHRSPaS001692

SHRSPaS001876

SHRSPaS005289

SHRSPaS001228

SHRSPaS004991

SHRSPaS001648

SHRSPaS006707

SHRSPaS004231

SHRSPaS002720
36.902

36.902

37.341

38.351

38.957

39.564

40.161

40.166

41.749

42.521

43.23

43.821

43.975

46.137

46.137

47.827

48.717

49.813

50.341

51.049

51.331

52.199

52.367

52.927

54.8

54.8

56.59

56.735

58.118

58.653

58.916

60.118

60.78

60.78

62.226

62.88

63.385

63.385

65.632

0

0.664

0.773

2.409

3.195

4.397

4.891

7.541

$\mathbf{8 . 8 0 3}$ F

$9.796 \mathrm{~F}$

$11.626 \mathrm{~F}$

15.671 F

$17.08 \mathrm{~F}$

18.179 F

$19.193 \mathrm{~F}$

$19.909 \mathrm{~F}$

23.273 F

Continued next page 
Supplemental Table 3. Continued.

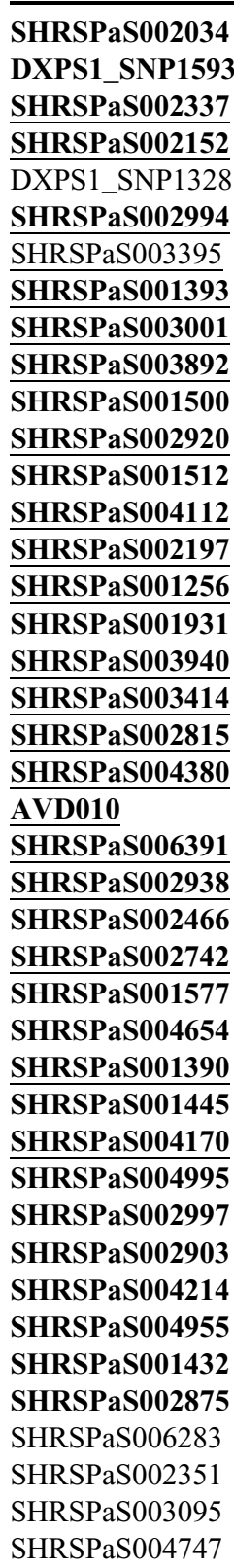

group 11

SHRSPa203

SHRSPaS003442

AVD022

SHRSPaS003135

SHRSPaS002683

AVT448

SHRSPaS003783

SHRSPaS002750

SHRSPaS001233

SHRSPaS004529

SHRSPaS002839

SHRSPaS004285

SHRSPaS004920

SHRSPaS005726

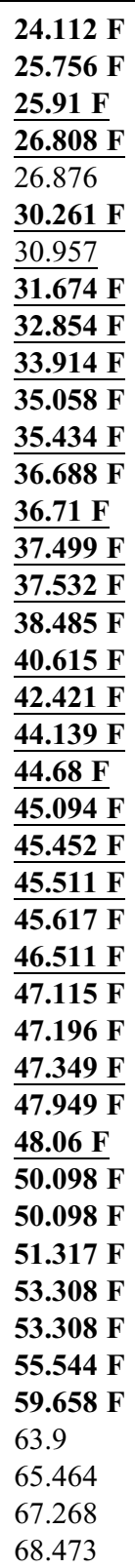

$$
\begin{gathered}
-15.593 \\
0 \\
3.311 \\
4.039 \\
6.661 \\
6.884 \\
8.863 \\
9.273 \\
10.35 \\
10.421 \\
11.257 \\
12.698 \\
13.306 \\
14.805
\end{gathered}
$$

\begin{tabular}{|c|c|}
\hline SHRSPaS003476 & 15.75 \\
\hline SHRSPaS001709 & 15.75 \\
\hline SHRSPaS001235 & 16.485 \\
\hline SHRSPaS001977 & 18.371 \\
\hline AUCR202 & 19.875 \\
\hline SHRSPaS006118 & 20.982 \\
\hline SHRSPaS001494 & 21.015 \\
\hline$\underline{\text { SHRSPaS004272 }}$ & $21.992 \mathrm{CP}$ \\
\hline$\overline{\text { SHRSPaS004238 }}$ & 23.552 \\
\hline SHRSPaS002263 & 24.169 \\
\hline SHRSPaS004983 & 24.275 \\
\hline AVD116 & 25.043 \\
\hline$\underline{\text { SHRSPaS004232 }}$ & $\underline{25.151 \mathrm{CP}}$ \\
\hline VTC1_1121 & 26.8 \\
\hline VTC1_1084 & 27.267 \\
\hline VTC1_1187 & 28.072 \\
\hline SHRSPaS001665 & 29.627 \\
\hline SHRSPaS001649 & 29.65 \\
\hline SHRSPaS002491 & 29.789 \\
\hline SHRSPaS003895 & 30.222 \\
\hline SHRSPaS002621 & 30.634 \\
\hline SHRSPaS001260 & 30.855 \\
\hline SHRSPaS006777 & 31.904 \\
\hline SHRSPaS003138 & 32.112 \\
\hline SHRSPaS006702 & 32.209 \\
\hline SHRSPaS001151 & 32.761 \\
\hline SHRSPaS004039 & 32.761 \\
\hline$\underline{\text { SHRSPaS002602 }}$ & $\underline{33.055 \mathrm{~A}}$ \\
\hline M1022 & 34.256 \\
\hline$\underline{\text { SHRSPaS002545 }}$ & $\underline{34.413 \mathrm{~A}}$ \\
\hline$\overline{\text { SHRSPaS003786 }}$ & 34.81 \\
\hline$\underline{\text { SHRSPaS001352 }}$ & $\underline{34.984 \mathrm{~A}}$ \\
\hline$\overline{\text { SHRSPaS003977 }}$ & $\overline{37.506}$ \\
\hline SHRSPaS004049 & 37.609 \\
\hline SHRSPaS001989 & 38.343 \\
\hline SHRSPaS002813 & 38.474 \\
\hline SHRSPaS003082 & 39.352 \\
\hline SHRSPaS003374 & $40.396 \mathrm{~A}$ \\
\hline$\overline{\text { SHRSPaS001213 }}$ & 41.746 \\
\hline SHRSPaS005008 & 42.21 \\
\hline SHRSPaS002803 & 42.863 \\
\hline SHRSPaS002011 & $43.178 \mathrm{~A}$ \\
\hline$\overline{\text { SHRSPaS004427 }}$ & $\overline{44.109 \mathrm{~A}}$ \\
\hline$\overline{\text { SHRSPaS002807 }}$ & 45.033 \\
\hline SHRSPaS001789 & 46.037 \\
\hline SHRSPaS002403 & 46.57 \\
\hline SHRSPaS001429 & 47.204 \\
\hline SHRSPaS001122 & 47.655 \\
\hline SHRSPaS001120 & 49.289 \\
\hline SHRSPaS002895 & 49.913 \\
\hline SHRSPaS001234 & 54.212 \\
\hline SHRSPaS002438 & 54.212 \\
\hline SHRSPaS003304 & 54.86 \\
\hline SHRSPaS001317 & 55.615 \\
\hline SHRSPaS002265 & 55.849 \\
\hline SHRSPaS002328 & 56.457 \\
\hline SHRSPaS002588 & 57.347 \\
\hline SHRSPaS002038 & 57.903 \\
\hline SHRSPaS003497 & 57.913 \\
\hline
\end{tabular}

Supplemental Table 3. Continued.

Continued next page 
Supplemental Table 3. Continued.

SHRSPaS002609
SHRSPaS001388
SHRSPaS003428
AVT001
SHRSPaS004508
SHRSPaS004295
SHRSPaS004625
SHRSPaS003928
SHRSPaS004177
SHRSPaS003479
SHRSPaS003327
SHRSPaS003317
SHRSPaS001802
SHRSPaS002719
SHRSPaS001650
SHRSPaS001745
SHRSPaS001270
PSY_SNP629or945
SHRSPaS003110
SHRSPaS001623
SHRSPaS002303
SHRSPaS003888
SHRSPaS001863
SHRSPaS001328
SHRSPaS003180
SHRSPaS001815
PSY_SNP370or686
SHRSPaS001643
SHRSPaS006056
SHRSPaS003207

group 12

\begin{tabular}{ll} 
SHRSPaS003393 & 0 \\
SHRSPaS003248 & 2.081 \\
AVD117 & 2.89 \\
SHRSPaS002662 & 4.505 \\
SHRSPaS003402 & 4.505 \\
SHRSPaS003265 & 6.588 \\
SHRSPaS001356 & $\underline{8.656 \mathrm{~B}}$ \\
\hline SHRSPaS001322 & $\underline{10.728 \mathrm{~B}}$ \\
\hline SHRSPaS005017 & $\underline{12.781 \mathrm{~B}}$ \\
\hline SHRSPaS003368 & $\underline{14.855 \mathrm{~B}}$ \\
\hline SHRSPaS002902 & $\underline{14.855 \mathrm{~B}}$ \\
\hline AVT386 & $\underline{16.374 \mathrm{~B}}$ \\
\hline SHRSPaS003965 & 17.445 \\
SHRSPaS003179 & 19.447 \\
SHRSPaS002003 & 19.447 \\
SHRSPaS001513 & $\underline{21.643 \mathrm{H}}$ \\
SHRSPaS001792 & 24.593 \\
SHRSPaS006852 & 25.916 \\
SHRSPaS005416 & 25.966 \\
SHRSPaS002243 & 26.645 \\
SHRSPaS003434 & 27.133 \\
SHRSPaS001655 & 27.961 \\
SHRSPaS004584 & 28.702 \\
SHRSPaS002624 & 29.09 \\
SHRSPaS005587 & 29.971 \\
SHRSPaS003320 & 30.136 \\
&
\end{tabular}

Continued next page
AVD117

SHRSPaS0032

SHRSPaS001356

AVT386

SHRSPaS003965

SHRSPaS003179

SHRSPaS001792

SHRSPaS006852

SHRSPaS001655

SHRSPaS003320
Supplemental Table 3. Continued.
58.345

58.538

58.719

59.309

59.726

60.143

60.53

61.52

61.856

62.278

62.278

63.21

63.21

63.668

64.337

64.852

$\frac{65.225 \mathrm{~A}}{67.225}$

67.335

69.225

70.506

71.047

71.71

72.389

72.64

73.147

73.956

75.612

78.049

80.349

\begin{tabular}{ll}
\hline SHRSPaS002331 & 30.94 \\
AVD015 & 32.143 \\
SHRSPaS002307 & 33.669 \\
SHRSPaS001125 & 35.166 \\
SHRSPaS004126 & 36.183 \\
SHRSPaS002194 & 37.07 \\
SHRSPaS001861 & 38.751 \\
SHRSPaS002010 & 38.896 \\
SHRSPaS001170 & 38.941 \\
SHRSPaS005010 & 38.956 \\
SHRSPaS001946 & 39.443 \\
SHRSPaS001754 & 39.858 \\
SHRSPaS004103 & 39.924 \\
SHRSPaS002339 & 40.529 \\
SHRSPaS002145 & 41.25 \\
AVD044 & 41.729 \\
SHRSPaS001706 & 41.848 \\
SHRSPaS001941 & 42.528 \\
SHRSPaS001455 & 42.954 \\
AUCR017 & 43.91 \\
SHRSPaS003189 & 45.218 \\
SHRSPaS003659 & 46.144 \\
SHRSPaS003946 & 46.473 \\
SHRSPaS002231 & 48.098 \\
SHRSPaS002995 & 50.081 \\
SHRSPaS001744 & 51.89 \\
SHRSPaS006854 & 53.504 \\
SHRSPaS001194 & $55.852 \mathrm{~A}$ \\
\hline SHRSPaS002854 & 66.236 \\
SHRSPaS003716 & $68.354 \mathrm{~T}$ \\
\hline
\end{tabular}

